\title{
K-theoretic Donaldson-Thomas theory and the Hilbert scheme of points on a surface
}

\author{
Noah Arbesfeld
}

\begin{abstract}
Integrals of characteristic classes of tautological sheaves on the Hilbert scheme of points on a surface frequently arise in enumerative problems. We use the K-theoretic Donaldson-Thomas theory of certain toric Calabi-Yau threefolds to study K-theoretic variants of such expressions.

We study limits of the K-theoretic Donaldson-Thomas partition function of a toric Calabi-Yau threefold under certain one-parameter subgroups called slopes and formulate a condition under which two such limits coincide. We then explicitly compute the limits of components of the partition function under so-called preferred slopes, obtaining explicit combinatorial expressions related to the refined topological vertex of Iqbal, Kosçaz and Vafa.

Applying these results to specific Calabi-Yau threefolds, we deduce dualities satisfied by a generating function built from tautological bundles on the Hilbert scheme of points on $\mathbb{C}^{2}$. We then use this duality to study holomorphic Euler characteristics of exterior and symmetric powers of tautological bundles on the Hilbert scheme of points on a general surface.
\end{abstract}

\section{Introduction}

\subsection{Tautological classes on the Hilbert scheme of points on a surface}

Let $S$ be a complex smooth quasi-projective surface, and let $S^{[n]}$ denote the Hilbert scheme of $n$ points on $S$. The moduli space $S^{[n]}$ is a smooth quasi-projective scheme of dimension $2 n$ parametrizing zero-dimensional length $n$ subschemes $Y \subset S$ or, equivalently, ideal sheaves $\mathcal{I}_{Y} \subset \mathcal{O}_{S}$ of colength $n$.

Given a line bundle $\mathcal{L}$ on $S$, one can use the universal family $\Sigma_{n} \subset S^{[n]} \times S$ as a correspondence to produce a rank $n$ vector bundle $\mathcal{L}^{[n]}$ on $S^{[n]}$. Namely, if $p_{S^{[n]}}: S^{[n]} \times S \rightarrow S^{[n]}$ and $p_{S}: S^{[n]} \times S \rightarrow S$ are the projection maps, we set

$$
\mathcal{L}^{[n]}=\left(p_{S[n]}\right)_{*}\left(\mathcal{O}_{\Sigma_{n}} \otimes p_{S}^{*} \mathcal{L}\right)
$$

Received 2 November 2020, accepted in final form 11 February 2021.

2020 Mathematics Subject Classification 14C05, 14N35, $14 \mathrm{C} 35$.

Keywords: Hilbert schemes, Donaldson-Thomas theory.

This journal is (C) Foundation Compositio Mathematica 2021. This article is distributed with Open Access under the terms of the Creative Commons Attribution Non-Commercial License, which permits non-commercial reuse, distribution, and reproduction in any medium, provided that the original work is properly cited. For commercial re-use, please contact the Foundation Compositio Mathematica.

This work was supported in part by the Department of Defense (DoD) through the National Defense Science and Engineering Graduate Fellowship (NDSEG) Program, by the National Science Foundation under Grant No. DMS-1440140 while the author was in residence at the Mathematical Sciences Research Institute in Berkeley, CA during the Spring 2018 semester and by EPSRC grant EP/R013349/1. 


\section{N. Arbesfeld}

the vector bundle $\mathcal{L}^{[n]}$ is called a tautological bundle, and its fiber over a subscheme $Y \in S^{[n]}$ is $H^{0}\left(\mathcal{O}_{Y} \otimes \mathcal{L}\right)$. Characteristic classes of tautological bundles encode geometric information. For example, series of the form

$$
\sum_{i, j, n \geqslant 0} m^{i} y^{j} z^{n} \int_{S[n]} c_{i}\left(\mathcal{L}^{[n]}\right) s_{j}\left(\mathcal{L}^{[n]}\right)
$$

arise in [MOP17] in the study of the cohomology ring of the moduli space of $K 3$ surfaces. The special case

$$
\sum_{n \geqslant 0} z^{n} \int_{S[n]} s_{2 n}\left(\mathcal{L}^{[n]}\right)
$$

is the subject of recent activity; its terms count secants to the projective embedding of $S$ given by $\mathcal{L}$ and are relevant to the computation of certain Donaldson invariants on $S$. The precise form of this series was conjectured by Lehn in [Leh99] and was recently proved, first for $S$ with $\mathcal{K}_{S}$ numerically trivial by Marian, Oprea and Pandharipande in [MOP17] and subsequently for general $S$ by Voisin and by Marian, Oprea and Pandharipande (and, independently, by Szenes and Vergne) in [Voi19, MOP19].

We study K-theoretic versions of series akin to (1.1). For a vector bundle $\mathcal{V}$ on a scheme $X$, set

$$
\Lambda_{m}^{\bullet} \mathcal{V}=\sum_{i \geqslant 0}^{\infty}(-m)^{i} \Lambda^{i} \mathcal{V} \in K(X)[[m]], \quad \operatorname{Sym}_{y}^{\bullet} \mathcal{V}=\sum_{i \geqslant 0}^{\infty} y^{i} \operatorname{Sym}^{i} \mathcal{V} \in K(X)[[y]]
$$

Then, a K-theoretic version of (1.1) is

$$
\sum_{n \geqslant 0} z^{n} \chi\left(S^{[n]}, \Lambda_{m}^{\bullet}\left(\mathcal{L}^{[n]}\right) \otimes \operatorname{Sym}_{y}^{\bullet}\left(\mathcal{L}^{[n]}\right)\right)
$$

Given an Euler characteristic $\chi(\mathscr{M}, \mathcal{F})$ of a torus-equivariant coherent sheaf $\mathcal{F}$ on a quasiprojective scheme $\mathscr{M}$ equipped with torus action, we identify $\chi(\mathscr{M}, \mathcal{F})$ with its character as a torus representation. For all examples we consider, such characters are rational functions on the torus.

1.1.1 Universal series. A general framework is developed in [EGL01] for analyzing expressions of a form similar to (1.1) and (1.4) using algebraic cobordism. To be precise, let

$$
\psi_{1}(x) \in 1+x \mathbb{Q}\left[y_{j}^{1}\right][[x]], \quad \psi_{2}(x) \in \mathbb{Q}\left[y_{j}^{2}\right][[x]]
$$

be power series with coefficients in some polynomial rings $\mathbb{Q}\left[y_{j}^{1}\right]$ and $\mathbb{Q}\left[y_{j}^{2}\right]$. For a vector bundle $\mathcal{V}$ with Chern roots $v_{i}$, set

$$
\Psi_{1}(\mathcal{V})=\prod_{i} \psi_{1}\left(v_{i}\right), \quad \Psi_{2}(\mathcal{V})=\prod_{i} \psi_{2}\left(v_{i}\right)
$$

Set

$$
\Psi(S, \mathcal{L})=\sum_{n \geqslant 0} z^{n} \int_{S^{[n]}} \Psi_{1}\left(\mathcal{L}^{[n]}\right) \Psi_{2}\left(\mathcal{T} S^{[n]}\right) .
$$

Then [EGL01, Theorem 4.2] implies that there exist $A_{1}, \ldots, A_{4} \in \mathbb{Q}[[z]]$ such that

$$
\Psi(S, \mathcal{L})=\exp \left(c_{1}(\mathcal{L})^{2} A_{1}+c_{1}(\mathcal{L}) c_{2}(S) A_{2}+c_{1}(S)^{2} A_{3}+c_{2}(S) A_{4}\right)
$$

for all pairs $(S, \mathcal{L})$. 


\section{K-THEORETIC DT THEORY AND HilBerT SCHEMES}

1.1.2 Localization expressions. The series $A_{i}$ can be determined by the values of $\Psi(S, \mathcal{L})$ for toric $S$. One approach to studying such values is to use equivariant localization to further reduce to an equivariant analog of (1.5) where $S$ is $\mathbb{C}^{2}$ equipped with the action of $\left(\mathbb{C}^{*}\right)^{2}=\operatorname{diag}\left(t_{1}, t_{2}\right)$ and $\mathcal{L}$ is an equivariant twist of the trivial bundle.

The torus action on $\mathbb{C}^{2}$ lifts to an action on $\left(\mathbb{C}^{2}\right)^{[n]}$. The torus-fixed points of $\left(\mathbb{C}^{2}\right)^{[n]}$ correspond to monomial ideals $I_{\lambda} \subset \mathbb{C}\left[z_{1}, z_{2}\right]$ of colength $n$, indexed by (two-dimensional) partitions $\lambda$ of size $n$. Our notation for partitions is set in Section 1.4. Here

$$
I_{\lambda}=\operatorname{Span}\left\{z_{1}^{b_{1}} z_{2}^{b_{2}} \mid\left(b_{1}, b_{2}\right) \notin \lambda\right\} .
$$

Denote by $T_{\lambda}$ the torus character of the tangent space to $I_{\lambda} \in\left(\mathbb{C}^{2}\right)^{[n]}$. It was shown in $[\mathrm{ES} 87$, Lemma 3.2] that $T_{\lambda}$ admits a combinatorial formula in terms of the arm lengths $a(\square)$ and leg lengths $l(\square)$ of boxes in the corresponding partition:

$$
T_{I_{\lambda}}\left(\mathbb{C}^{2}\right)^{[n]}=\sum_{\square \in \lambda} t_{1}^{-l(\square)} t_{2}^{a(\square)+1}+t_{1}^{l(\square)+1} t_{2}^{-a(\square)} .
$$

The torus characters of fibers of tautological bundles over the fixed points of the Hilbert scheme are also described combinatorially. Let $\mathcal{O}(l)$ denote the trivial line bundle on $\mathbb{C}^{2}$ twisted by the torus weight $l$. We have

$$
\left.(\mathcal{O}(l))^{[n]}\right|_{I_{\lambda}}=\sum_{\square=\left(b_{1}, b_{2}\right) \in \lambda} l t_{1}^{-b_{1}} t_{2}^{-b_{2}} .
$$

By equivariant localization, expressions of the form (1.5) may be written in terms of the combinatorics of partitions. This approach has been used in similar settings to compute universal series up to some given order; see for example [GK20, GK19]. However, some structures of such universal series may be intractable from this perspective. For example, it is not known how to use these equivariant methods to determine the series (1.2) studied in [MOP17, Voi19, MOP19].

1.1.3 Symmetries of generating functions. In this paper, we find identities for certain tautological classes over the Hilbert scheme of points on $\mathbb{C}^{2}$. Introduce formal variables $z, y, m_{1}, m_{2}$ and $m_{3}$, and consider the following expression assembled from exterior and symmetric powers of tautological bundles.

Definition 1.1. Set $F\left(z, m_{1}, m_{2}, m_{3}, y\right)\left(t_{1}, t_{2}\right)$ to be the series of torus-equivariant Euler characteristics

$$
\sum_{n \geqslant 0}(-z)^{n} \chi\left(\left(\mathbb{C}^{2}\right)^{[n]}, \Lambda_{m_{1}}^{\bullet}\left(\mathcal{O}^{[n]}\right)^{\vee} \otimes \Lambda_{m_{2}}^{\bullet}\left(\mathcal{O}^{[n]}\right)^{\vee} \otimes \Lambda_{m_{3} y}^{\bullet} \mathcal{O}^{[n]} \otimes \operatorname{Sym}_{y}^{\bullet} \mathcal{O}^{[n]} \otimes \Lambda^{n} \mathcal{O}^{[n]}\right)
$$

By K-theoretic equivariant localization [Tho92, Theorem 3.5],

$$
\begin{aligned}
& F\left(z, m_{1}, m_{2}, m_{3}, y\right)\left(t_{1}, t_{2}\right) \\
& \quad=\sum_{\lambda} \frac{z^{|\lambda|}}{\Lambda^{\bullet}\left(T_{\lambda}^{\vee}\right)} \prod_{\left(b_{1}, b_{2}\right) \in \lambda} \frac{\left(1-m_{1} t_{1}^{b_{1}} t_{2}^{b_{2}}\right)\left(1-m_{2} t_{1}^{b_{1}} t_{2}^{b_{2}}\right)\left(1-m_{3} y t_{1}^{-b_{1}} t_{2}^{-b_{2}}\right)}{y-t_{1}^{b_{1}} t_{2}^{b_{2}}} ;
\end{aligned}
$$

here, given a torus character $V=\sum_{i} u_{i}$, where each $u_{i}$ is a torus weight, we set

$$
\Lambda^{\bullet} V=\prod_{i}\left(1-u_{i}\right)
$$




\section{N. Arbesfeld}

We regard $F$ as a power series in the variables $y$ and $z$ whose coefficients are rational functions in the remaining variables $m_{1}, m_{2}, m_{3}, t_{1}$ and $t_{2}$.

We show that after normalization, the series $F$ enjoys two types of symmetries: one between the "box-counting variable" $z$ and the "Segre variable" $y$, and another between the "Chern variables" $m_{1}, m_{2}, m_{3}$.

TheOREM 1.2. We have

$$
\frac{F\left(z, m_{1}, m_{2}, m_{3}, y\right)\left(t_{1}, t_{2}\right)}{F\left(z, m_{1}, m_{2}, m_{3}, 0\right)\left(t_{1}, t_{2}\right)}=\frac{F\left(z, m_{1}, m_{3}, m_{2}, y\right)\left(t_{1}, t_{2}\right)}{F\left(z, m_{1}, m_{3}, m_{2}, 0\right)\left(t_{1}, t_{2}\right)}=\frac{F\left(y, m_{1}, m_{2}, m_{3}, z\right)\left(t_{1}, t_{2}\right)}{F\left(y, m_{1}, m_{2}, m_{3}, 0\right)\left(t_{1}, t_{2}\right)}
$$

Moreover, we show that the denominators appearing in (1.8) can be characterized using the plethystic exponential.

Proposition 1.3. We have

$$
F\left(z, m_{1}, m_{2}, m_{3}, 0\right)\left(t_{1}, t_{2}\right)=\exp \left(\sum_{n \geqslant 1}-\frac{z^{n}}{n} \frac{\left(1-m_{1}^{n}\right)\left(1-m_{2}^{n}\right)}{\left(1-t_{1}^{-n}\right)\left(1-t_{2}^{-n}\right)}\right) .
$$

Note that the $n=1$ term of the sum in the argument on the right-hand side of (1.9) is the $z^{1}$-term of the left-hand side. We remark that Proposition 1.3 is also a consequence of [WZ14, Theorem 3.2]. Mellit has observed that certain specializations of Theorem 1.2 can also be deduced from the combinatorics of Macdonald polynomials; see [Mel16, Section 7], where such expressions are studied in connection with mixed Hodge polynomials of character varieties.

1.1.4 Applications to tautological bundles. Theorem 1.2 and Proposition 1.3 can be used to study the holomorphic Euler characteristics of exterior powers, and certain symmetric powers, of tautological bundles $\mathcal{L}^{[n]}$ on $S^{[n]}$ for general surfaces $S$. For example, we prove the following.

Corollary 1.4. If $\chi\left(\mathcal{O}_{S}\right)=1$, then for $n \geqslant k$, we have

$$
\chi\left(S^{[n]}, \operatorname{Sym}^{k}\left(\mathcal{L}^{[n]}\right)\right)=\left(\begin{array}{c}
\chi(\mathcal{L})+k-1 \\
k
\end{array}\right) .
$$

Theorem 1.2 and Proposition 1.3 also control holomorphic Euler characteristics of exterior powers of tautological bundles induced from rank two vector bundles on $S$. They also encode families of nontrivial combinatorial identities. For example, it is evident that, for $l>n$, the coefficient of $z^{n} m_{1}^{l}$ is 0 on the left-hand side of (1.8), but this is not obvious on the right-hand side.

\subsection{K-theoretic enumerative geometry}

We deduce Theorem 1.2 and Proposition 1.3 from equalities of particular limits of K-theoretic Donaldson-Thomas (DT) partition functions of certain Calabi-Yau threefolds.

These results follow from a more general framework that produces identities involving generating series called K-theoretic Nekrasov partition functions; these are series formed from Euler characteristics of tautological bundles over instanton moduli spaces. Many invariants of geometric interest can be phrased in terms of K-theoretic Nekrasov functions and their cohomological analogs; examples include the K-theoretic Vafa-Witten invariants studied in [Tho20] and the elliptic genera of moduli spaces of sheaves studied in [GK19].

Our approach is based on two observations. First, it is shown in [NO16, Section 8] that special limits of the K-theoretic DT partition function can be expressed in terms of the refined topological 


\section{K-THEORETiC DT THEORY AND Hilbert SCHEMES}

vertex of [IKV09]. Second, it is shown in the physics literature on "geometric engineering" that particular computations using the refined topological vertex can be matched with the K-theoretic Nekrasov partition functions of certain gauge theories; some examples of this phenomenon are computed in [IKV09, Tak08].

We now outline this approach in more detail.

Let $X$ be a complex smooth threefold, and let $\mathrm{DT}(X)$ denote the Hilbert scheme of curves in $X$. In [NO16], Nekrasov and Okounkov introduce the K-theoretic Donaldson-Thomas partition function $Z_{\mathrm{DT}}(X)$, a generating series formed from Euler characteristics

$$
\chi\left(\operatorname{DT}(X, \beta, n), \tilde{\mathcal{O}}^{\text {vir }}\right) .
$$

Here $\operatorname{DT}(X, \beta, n)$ is a component of the Hilbert scheme of curves equipped with the symmetric perfect obstruction theory arising from its realization as a moduli space of ideal sheaves, and $\tilde{\mathcal{O}}^{\text {vir }}$ is a certain modification of the virtual structure sheaf.

Our applications will be in the case when $X$ is toric Calabi-Yau and the partition function $Z_{\mathrm{DT}}(X)$ is formed from torus-equivariant Euler characteristics.

1.2.1 Slope independence. Given a complex torus $\mathbb{T}$, a power series $Z$ with coefficients in $\mathbb{Q}(\mathbb{T})$ and a one-parameter subgroup $\sigma: \mathbb{C}^{\times} \rightarrow \mathbb{T}$, define $Z^{\sigma}$ to be the power series (again with coefficients in $\mathbb{Q}(\mathbb{T})$ ) given by

$$
Z^{\sigma}(\bar{t})=\lim _{z \rightarrow 0} Z(\sigma(z) \bar{t})
$$

for $\bar{t} \in \mathbb{T}$ if the limit in question exists.

Given such a one-parameter subgroup $\sigma$ and a weight $w: \mathbb{T} \rightarrow \mathbb{C}^{\times}$, there exists some integer $r$ such that $w(\sigma(z) \bar{t})=z^{r} w(\bar{t})$ for all $z \in \mathbb{C}^{\times}, \bar{t} \in \mathbb{T}$. Assume that this $r$ is nonzero. We say that $w$ is attracting with respect to $\sigma$ if $r>0$, and repelling with respect to $\sigma$ if $r<0$.

It is shown in [NO16, Proposition 7.4] that for certain $Z$ formed from Euler characteristics over proper stable pair moduli spaces on toric Calabi-Yau threefolds, the limits $Z^{\sigma}$ are independent of the choice of generic $\sigma$ landing in the Calabi-Yau subtorus.

We are interested, however, in such limits of $Z_{\mathrm{DT}}(X)$. As the Hilbert scheme of curves in a noncompact threefold has noncompact components, we require a version of [NO16, Proposition 7.4] for noncompact geometries. In particular, let $M$ be a component $\operatorname{DT}(X, \beta, n)$ of the Hilbert scheme of curves in a smooth toric Calabi-Yau threefold $X$. Let $T$ denote the threedimensional torus acting on $X$, and let $\kappa$ be the $T$-weight of the anticanonical bundle $\mathcal{K}_{X}^{\vee}$.

A slope is a one-parameter subgroup $\sigma: \mathbb{C}^{\times} \rightarrow \operatorname{ker}(\kappa) \subset T$. Call a weight $w \in T^{\vee}$ a noncompact direction of $X$ if the fixed locus $(\mathrm{DT}(X))^{\operatorname{ker}(w)}$ has a nonproper component. We prove the following.

THEOREM 1.5. If two generic slopes $\sigma_{1}$ and $\sigma_{2}$ share the same attracting or repelling behavior with respect to each noncompact direction of $X$, then

$$
\chi\left(M, \tilde{\mathcal{O}}^{\text {vir }}\right)^{\sigma_{1}}=\chi\left(M, \tilde{\mathcal{O}}^{\text {vir }}\right)^{\sigma_{2}} \in \mathbb{Q}\left[\kappa^{1 / 2}\right] .
$$

1.2.2 Vertex and edge contributions. The next step is to explicitly compute limits of $Z_{\mathrm{DT}}(X)^{\sigma}$ for particular $X$ and $\sigma$. For toric threefolds $X$, the partition function $Z_{\mathrm{DT}}(X)$ can be studied using equivariant localization. As shown in [MNOP06], the $T$-fixed points of $\mathrm{DT}(X)$ are given by configurations of three-dimensional partitions along the one-skeleton of the toric polytope $\Delta(X)$; the partition function can be written in terms of contributions from the vertices and edges of 
the one-skeleton. The edge contributions admit an explicit description in terms of $\left(\mathbb{C}^{2}\right)^{[n]}$; the contribution of the relevant edge configurations are given in Propositions 4.2 and 4.3.

The vertex contributions $V(\lambda, \mu, \nu)$ are more complicated. Their cohomological analogs are studied using the topological vertex; see [AKMV05, ORV06]. In K-theory, it is difficult to analyze $V(\lambda, \mu, \nu)$ in full generality. However, for generic $\sigma$, the resulting limit $V(\lambda, \mu, \nu)^{\sigma}$ simplifies: the equivariant parameters of $T$ enter this limit only through the character $\kappa^{1 / 2}$.

Moreover, it was explained in [NO16, Section 8] that for certain slopes $\sigma$ called preferred slopes (roughly, those that nearly preserve a coordinate direction in each toric chart of $X$ ), the limit $V(\lambda, \mu, \nu)^{\sigma}$ is related to the refined topological vertex of [IKV09]. Such limits are well suited to computation. In Proposition 4.9, we explicate the precise relationship between such $V(\lambda, \mu, \nu)^{\sigma}$ and the refined topological vertex. In particular, the choice of direction of a preferred slope corresponds to a choice of preferred direction of the refined topological vertex.

Theorem 1.5 may therefore be regarded as a mathematical formulation of the independence of the refined topological partition function of toric Calabi-Yau threefolds $X$ under certain choices of preferred direction; examples of this independence have been computed in [AK13, IKV09]. We note, however, that limits $Z_{\mathrm{DT}}(X)^{\sigma}$ often exhibit some dependence on $\sigma$; in other words, the refined topological partition function of a threefold $X$ can depend on the choice of preferred direction. This phenomenon can be seen, for example, in [IKV09, Section 5.3].

In the physics literature, computations using the refined topological vertex incorporate different so-called "framing factors"; see for instance [IKV09] and [Tak08]. The computation using DT invariants provides one consistent way to choose such factors.
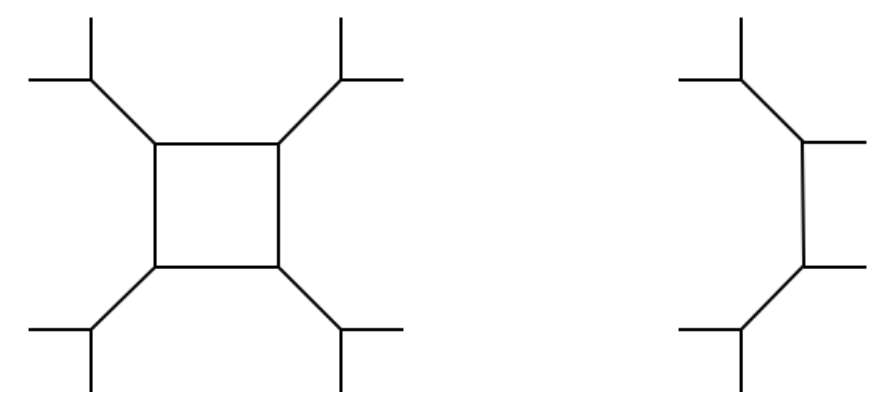

Figure 1: The toric geometries that give rise to Theorem 1.2 and Proposition 1.3, respectively

1.2.3 Limits of DT partition functions. We use these vertex and edge computations to compute $Z_{\mathrm{DT}}(X)^{\sigma_{1}}$ and $Z_{\mathrm{DT}}(X)^{\sigma_{2}}$, where $X$ is one of the Calabi-Yau threefolds whose toric skeleta are given in Figure 1, and $\sigma_{1}$ and $\sigma_{2}$ are two preferred slopes satisfying the hypotheses of Theorem 1.5. After a certain specialization, we deduce Theorem 1.2 and Proposition 1.3.

When applied to threefolds with more complicated toric diagrams, this approach will produce more complicated identities involving higher-rank Nekrasov functions. For example, a new feature that should emerge is a relationship between tautological integrals over $\operatorname{Hilb}\left(\mathbb{C}^{2}\right)$ and those over moduli spaces of higher-rank sheaves.

\subsection{Outline}

In Section 2, we define K-theoretic Donaldson-Thomas invariants of Calabi-Yau threefolds. In Section 3 , we prove a general relationship between noncompact directions in a moduli space and 


\section{K-THEORETIC DT THEORY AND HilBerT SCHEMES}

the limits of Euler characteristics of coherent sheaves under slopes in order to deduce Theorem 1.5. In Section 4, we recall from [MNOP06, NO16, Oko19] the descriptions of the vertex and edge contributions to K-theoretic Donaldson-Thomas invariants of toric Calabi-Yau threefolds in terms of partitions and write down explicit combinatorial descriptions of their limits under preferred slopes. In Section 5, we apply these results to specific Calabi-Yau threefolds to deduce Theorem 1.2 and Proposition 1.3. We apply these results in Section 6 to tautological classes over the Hilbert scheme of points on a general surface.

\subsection{Notation for partitions}

A two-dimensional partition $\lambda$ is either the empty sequence $\varnothing$ or an ordered finite sequence $\left(\lambda_{i}\right)=\left(\lambda_{1}, \lambda_{2}, \ldots\right)$ of nonincreasing positive integers. Equivalently, it is a finite collection of ordered pairs $\left(b_{1}, b_{2}\right) \in \mathbb{Z}_{\geqslant 0}^{2}$ such that if $\left(b_{1}, b_{2}\right) \in \lambda$, then for any $b_{1}^{\prime}$, $b_{2}^{\prime}$ satisfying $0 \leqslant b_{1}^{\prime} \leqslant b_{1}$ and $0 \leqslant b_{2}^{\prime} \leqslant b_{2}$, we also have $\left(b_{1}^{\prime}, b_{2}^{\prime}\right) \in \lambda$; the bijection between these two notions is given by

$$
\left\{\left(\lambda_{i}\right)\right\} \mapsto\left\{\left(b_{1}, b_{2}\right) \mid b_{2} \leqslant \lambda_{b_{1}+1}-1\right\} .
$$

We define the size $|\lambda|$ of a partition $\lambda$ to be $\sum_{i} \lambda_{i}$, define the length of $\lambda$ to be the length of the sequence $\left(\lambda_{i}\right)$ and set $\|\lambda\|^{2}=\sum_{i}\left|\lambda_{i}\right|^{2}$.

We sometimes abbreviate an ordered pair $\left(b_{1}, b_{2}\right)$ by the symbol $\square$; we refer to such a square as a box of $\lambda$. We draw a two-dimensional partition as an upper-left justified configuration of boxes with $\lambda_{i}$ boxes in the $i$ th row from the top; see Figure 2.

We let $\lambda^{t}$ denote the conjugate partition of $\lambda$, that is,

$$
\lambda^{t}=\left\{\left(b_{2}, b_{1}\right) \mid\left(b_{1}, b_{2}\right) \in \lambda\right\} .
$$

For a box $\square=\left(b_{1}, b_{2}\right) \in \lambda$, we let $a(\square)$ and $l(\square)$ denote the arm and leg lengths of $\square$, that is

$$
a(\square)=\lambda_{b_{1}+1}-b_{2}-1, \quad l(\square)=\lambda_{b_{2}+1}^{t}-b_{1}-1 ;
$$

see Figure 2. When the meaning is clear, we abbreviate $a(\square)$ by $a$ and $l(\square)$ by $l$.

\begin{tabular}{|l|l|l|l|}
\hline$(0,0)$ & $(0,1)$ & $(0,2)$ & $(0,3)$ \\
\hline$(1,0)$ & $(1,1)$ & $(1,2)$ & $(1,3)$ \\
\cline { 1 - 2 }$(2,0)$ & $(2,1)$ & \multicolumn{2}{|c}{} \\
\cline { 1 - 2 }$(3,0)$ & \multicolumn{2}{|c}{} \\
\cline { 1 - 1 } & \multicolumn{2}{|c}{} \\
\end{tabular}

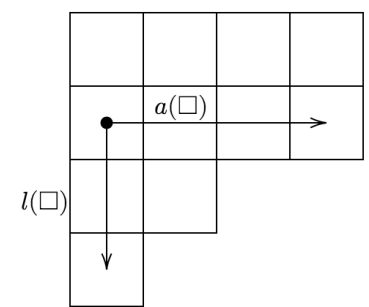

Figure 2: The two-dimensional partition $\lambda=(4,4,2,1)$ with boxes $\left(b_{1}, b_{2}\right)$ labeled. The box $\square=(1,0) \in \lambda$ has $a(\square)=3$ and $l(\square)=2$.

A three-dimensional partition $\pi$ is a (possibly infinite) collection of points of $\mathbb{Z}_{\geqslant 0}^{3}$ such that the following hold:

(i) If $\left(b_{1}, b_{2}, b_{3}\right) \in \pi$ and $0 \leqslant b_{i}^{\prime} \leqslant b_{i}$ for $i=1,2,3$, then $\left(b_{1}^{\prime}, b_{2}^{\prime}, b_{3}^{\prime}\right) \in \pi$.

(ii) There exists some integer $B_{\pi}$ such that, for any $\left(b_{1}, b_{2}, b_{3}\right) \in \pi$, at most one of the coordinates $b_{1}, b_{2}, b_{3}$ satisfies $b_{i}>B_{\pi}$. In other words, the asymptotics of $\pi$ along the coordinate directions of $\mathbb{R}_{\geqslant 0}^{3}$ are given by (finite, possibly empty) two-dimensional partitions. 


\section{N. Arbesfeld}

One can visualize such a partition as a collection of unit boxes in the octant $\mathbb{R}_{\geqslant 0}^{3}$ centered at the points $\left(b_{1}+\frac{1}{2}, b_{2}+\frac{1}{2}, b_{3}+\frac{1}{2}\right)$; see Figure 3 . Again, when clear, we abbreviate $\left(b_{1}, b_{2}, b_{3}\right)$ by the symbol $\square$.

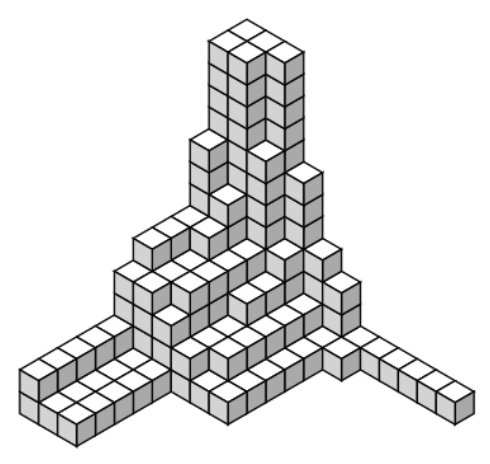

Figure 3: A three-dimensional partition $\pi$ with asymptotics $\pi^{(1)}=(2,1,1), \pi^{(2)}=(1)$ and $\pi^{(3)}=$ $(3,2)$

Given a three-dimensional partition $\pi$, let $\pi^{(k)}$ denote the two-dimensional partition describing the asymptotics of $\pi$ along the $k$ th coordinate axis; see Figure 3. Formally,

$$
\begin{aligned}
& \pi^{(1)}=\left\{\left(b_{1}, b_{2}\right) \mid\left(b, b_{1}, b_{2}\right) \in \pi \text { for all } b \geqslant 0\right\}, \\
& \pi^{(2)}=\left\{\left(b_{1}, b_{2}\right) \mid\left(b_{2}, b, b_{1}\right) \in \pi \text { for all } b \geqslant 0\right\}, \\
& \pi^{(3)}=\left\{\left(b_{1}, b_{2}\right) \mid\left(b_{1}, b_{2}, b\right) \in \pi \text { for all } b \geqslant 0\right\} .
\end{aligned}
$$

The ordering is chosen so that the two-dimensional partitions are compatible with a fixed choice of orientation on $\mathbb{R}_{\geqslant 0}^{3}$.

We let $\pi\langle k\rangle \subset \pi$ denote the infinite leg of the partition $\pi$ along the $k$ th coordinate axis. Formally,

$$
\begin{aligned}
& \pi\langle 1\rangle=\left\{\left(b, b_{1}, b_{2}\right) \mid\left(b_{1}, b_{2}\right) \in \pi^{(1)}, b \in \mathbb{Z}_{\geqslant 0}\right\}, \\
& \pi\langle 2\rangle=\left\{\left(b_{2}, b, b_{1}\right) \mid\left(b_{1}, b_{2}\right) \in \pi^{(2)}, b \in \mathbb{Z}_{\geqslant 0}\right\}, \\
& \pi\langle 3\rangle=\left\{\left(b_{1}, b_{2}, b\right) \mid\left(b_{1}, b_{2}\right) \in \pi^{(3)}, b \in \mathbb{Z}_{\geqslant 0}\right\} .
\end{aligned}
$$

\section{K-theoretic DT invariants}

Let $X$ be a smooth threefold. We impose the further assumption that $X$ is toric Calabi-Yau. In particular, $X$ is not projective. Let $T$ denote the three-dimensional torus $\operatorname{diag}\left(t_{1}, t_{2}, t_{3}\right)$ acting on $X$.

While this assumption has the advantage of simplifying the definition of K-theoretic Donaldson-Thomas invariants, it also obscures the generality in which these invariants can be formulated. One motivation for this definition is a conjectural connection with curve counting in associated Calabi-Yau fivefolds (see [NO16, Oko19].) Such a relationship is expected for any smooth threefold $X$. 


\section{K-THEORETIC DT THEORY AND HiLBERT SCHEMES}

\subsection{Donaldson-Thomas moduli space}

Let $\operatorname{DT}(X)$ denote the Hilbert scheme of curves in $X$. The moduli space $\operatorname{DT}(X)$ is a union of components $\operatorname{DT}(X, \beta, n)$ parametrizing projective subschemes $Y \subset X$ whose components are of dimension at most one with $[Y]=\beta$ and $\chi\left(\mathcal{O}_{Y}\right)=n$; here $[Y] \in H_{2}(X, \mathbb{Z})^{\text {eff }}$ denotes the class given by the one-dimensional components of $Y$ weighted by their multiplicities.

It is shown in [Tho00] that $\mathrm{DT}(X)$ admits another description as the moduli space of ideal sheaves: rank one, torsion-free sheaves $\mathcal{I}_{Y}$ on $X$ with trivial determinant. On the level of points, one identifies a subscheme $\mathcal{O}_{Y}$ with its ideal sheaf

$$
\mathcal{I}_{Y}=\operatorname{ker}\left(\mathcal{O}_{X} \rightarrow \mathcal{O}_{Y}\right)
$$

conversely, one passes from an ideal sheaf $\mathcal{I}_{Y}$ to the subscheme

$$
\mathcal{O}_{Y}=\operatorname{coker}\left(\mathcal{I}_{Y} \hookrightarrow\left(\mathcal{I}_{Y}\right)^{\vee \vee} \cong \mathcal{O}_{X}\right) \text {. }
$$

In contrast to Hilbert schemes of points on smooth surfaces, which are smooth, Hilbert schemes of curves on threefolds are generally of unknown dimension and highly singular. However, in [Tho00], Thomas used the ideal sheaf description to equip $\operatorname{DT}(X)$ with a $T$-equivariant symmetric perfect obstruction theory $E^{\bullet} \rightarrow \mathbb{L}_{\mathrm{DT}(X)}$ in the sense of Behrend-Fantechi (see [BF97]). By the constructions of, for example, [BF97, Remark 5.4], [CK09] and [Lee04, Section 2], the obstruction theory gives rise to the $T$-equivariant virtual sheaves in $K_{T}(\mathrm{DT}(X))$ needed for K-theoretic enumerative computations: the virtual structure sheaf $\mathcal{O}^{\text {vir }}$, the virtual tangent sheaf $\mathcal{T}^{\text {vir }}$ and the virtual canonical bundle $\mathcal{K}^{\mathrm{vir}}=\operatorname{det}\left(\mathcal{T}^{\mathrm{vir}}\right)^{\vee}$. In particular, from the obstruction theory of [Tho00], one concludes that

$$
\mathcal{T}_{\mathcal{I}}^{\text {vir }}=\operatorname{Def}_{\mathcal{I}}-\operatorname{Obs}_{\mathcal{I}}=\operatorname{Ext}^{1}(\mathcal{I}, \mathcal{I})-\operatorname{Ext}^{2}(\mathcal{I}, \mathcal{I}), \quad \mathcal{K}_{\mathcal{I}}^{\text {vir }}=\frac{\operatorname{det} \operatorname{Obs}_{\mathcal{I}}}{\operatorname{det} \operatorname{Def}_{\mathcal{I}}}
$$

As $X$ is Calabi-Yau, the anticanonical bundle $\mathcal{K}_{X}^{\vee}$ is a trivial bundle twisted by some $T$-weight $\kappa$. Serre duality implies that as $T$-characters, we have

$$
\operatorname{Def}_{\mathcal{I}}=\left(\operatorname{Obs}_{\mathcal{I}}\right)^{\vee} \cdot \kappa
$$

\subsection{Modified virtual structure sheaf}

The definition of K-theoretic Donaldson-Thomas invariants incorporates a twist of the virtual structure sheaf by a square root of the virtual canonical bundle. This modification endows the resulting invariants with certain symmetries that make for easier analysis. It also aligns the invariants with the indices of certain Dirac operators of interest in physics (see, for example, [Oko17, Section 3.2.7]). The invariants do not depend on the choice of square root.

The existence of such a square root is ensured by the following proposition.

Proposition 2.1 ([NO16, Section 6]). The line bundle $\mathcal{K}^{\text {vir }}$ admits a square root $\left(\mathcal{K}^{\mathrm{vir}}\right)^{1 / 2}$ in $\operatorname{Pic}(\operatorname{DT}(X))$. Moreover, if $\tilde{T}$ is a minimal cover of $T$ on which the square root $\kappa^{1 / 2}$ is defined, the line bundle $\left(\mathcal{K}^{\text {vir }}\right)^{1 / 2}$ carries a canonical $\tilde{T}$-equivariant structure.

We remark that in the special case when $X$ is Calabi-Yau, this proposition follows from an argument using Serre duality given in [NO16, Section 6.2.1]; such a statement is expected for general threefolds $X$ and is proved in [NO16, Section 6] when $\mathrm{DT}(X)$ is replaced by the moduli space $\mathrm{PT}(X)$ of stable pairs.

DeFinition 2.2. The modified virtual structure sheaf is

$$
\tilde{\mathcal{O}}^{\text {vir }}=\mathcal{O}^{\text {vir }} \otimes\left(\mathcal{K}^{\text {vir }}\right)^{1 / 2}
$$




\section{N. Arbesfeld}

\subsection{K-theoretic Donaldson-Thomas invariants}

Define the K-theoretic DT partition function to be

$$
Z_{\mathrm{DT}}(X)=\sum_{\substack{\beta \in H_{2}(X, \mathbb{Z})^{\mathrm{eff}} \\ n \in \mathbb{Z}}} Q^{n} u^{\beta} \chi\left(\mathrm{DT}(X, \beta, n), \tilde{\mathcal{O}}^{\mathrm{vir}}\right) \in \mathbb{Q}\left(t_{1}, t_{2}, t_{3}, \kappa^{1 / 2}\right)\left[\left[Q, Q^{-1}\right]\right]\left[u^{\beta}\right] .
$$

Given some fixed $\beta$, the space $\operatorname{DT}(X, \beta, n)$ is empty for $n \ll 0$.

It is convenient to normalize the Donaldson-Thomas partition function by the contribution from the Hilbert scheme of points on $X$; to this end, we define the reduced K-theoretic DT partition function to be

$$
Z_{\mathrm{DT}}^{\prime}(X)=\frac{Z_{\mathrm{DT}}(X)}{\sum_{n \in \mathbb{Z}} Q^{n} \chi\left(\mathrm{DT}(X, 0, n), \tilde{\mathcal{O}}^{\mathrm{vir}}\right)} \in \mathbb{Q}\left(t_{1}, t_{2}, t_{3}, \kappa^{1 / 2}\right)\left[\left[Q, Q^{-1}\right]\right]\left[u^{\beta}\right] .
$$

\subsection{The plethystic exponential}

Roughly speaking, the plethystic exponential takes as input the character of a virtual (possibly infinite-dimensional) representation $V$ of some group and, in the spirit of (1.3), outputs the character of

$$
\sum_{n \geqslant 0} \operatorname{Sym}^{n} V
$$

when this character is well defined. To simplify the exposition, we give a precise definition for the two settings in which we will use the plethystic exponential and refer the reader to [Mel16, Section 3] or [Oko17, Section 2.1] for an expanded discussion.

First, given formal variables $x_{1}, \ldots, x_{m}$ and finitely many Laurent monomials $u_{i}\left(x_{1}, \ldots, x_{m}\right)$ and $v_{j}\left(x_{1}, \ldots, x_{m}\right)$ such that no $u_{i}$ is equal to 1 , we define

$$
\operatorname{Sym}^{\bullet}\left(\sum_{i} u_{i}-\sum_{j} v_{j}\right)=\frac{\prod_{j} 1-v_{j}}{\prod_{i} 1-u_{i}} \in \mathbb{Q}\left(x_{1}, \ldots, x_{n}\right) .
$$

Second, this definition can be generalized so that $\mathrm{Sym}^{\bullet}$ takes as arguments certain series whose coefficients are rational functions. For example, let $z, x_{1}, \ldots, x_{m}, t_{1}, t_{2}$ be formal variables and $w_{1}, w_{2}$ be monomials in $t_{1}, t_{2}$. Given Laurent polynomials $f_{i}\left(x_{1}, \ldots, x_{m}, t_{1}, t_{2}\right)$ for $i>0$, define

$$
\begin{aligned}
\operatorname{Sym} & \left(\sum_{i \geqslant 1} z^{i} \frac{f_{i}\left(x_{1}, \ldots, x_{m}, t_{1}, t_{2}\right)}{\left(1-w_{1}\right)\left(1-w_{2}\right)}\right) \\
& =\exp \left(\sum_{i, n \geqslant 1} \frac{\left(z^{i}\right)^{n}}{n} \frac{f_{i}\left(x_{1}^{n}, \ldots, x_{m}^{n}, t_{1}^{n}, t_{2}^{n}\right)}{\left(1-w_{1}^{n}\right)\left(1-w_{2}^{n}\right)}\right) \in \mathbb{Q}\left[x_{1}^{ \pm}, \ldots, x_{m}^{ \pm}\right]\left(t_{1}, t_{2}\right)[[z]] .
\end{aligned}
$$

For example, the right-hand side of (1.9) can be rewritten as

$$
\operatorname{Sym}^{\bullet}\left(-z \frac{\left(1-m_{1}\right)\left(1-m_{2}\right)}{\left(1-t_{1}^{-1}\right)\left(1-t_{2}^{-1}\right)}\right) \text {. }
$$

Each of the definitions (2.2) and (2.3) can be formulated in the spirit of the other in a straightforward manner. It will be helpful to pass between these two interpretations in the calculations of Section 5.3. 


\section{K-THEORETIC DT THEORY AND HiLBERT SCHEMES}

\subsection{Equivariant localization}

We study K-theoretic invariants using virtual equivariant localization as developed in [FG10, GP99], [CK09] and [Qu18]; a concise exposition of the application we use can be found in [Tho20]. In this subsection, let $\mathscr{M}$ be a quasi-projective scheme equipped with the action of a torus $\mathbb{T}$ and a $\mathbb{T}$-equivariant perfect obstruction theory. Suppose that the fixed locus $\mathscr{M}^{\mathbb{T}}$ is compact (and nonempty). Let $\mathcal{F}$ be a $\mathbb{T}$-equivariant coherent sheaf on $\mathscr{M}$. Equivariant localization asserts, first, that

$$
\chi\left(\mathscr{M}, \mathcal{F} \otimes \mathcal{O}^{\text {vir }}\right) \in \mathbb{Q}\left[t_{k}^{ \pm}\right]\left[\frac{1}{1-u_{i}}\right],
$$

where the $u_{i}$ range over the set of $\mathbb{T}$-weights appearing in $N_{\mathscr{M} / \mathscr{M}^{\mathbb{T}}}^{\mathrm{vir}}$. Moreover, it expresses $\chi\left(\mathscr{M}, \mathcal{F} \otimes \mathcal{O}^{\text {vir }}\right)$ as an Euler characteristic over the fixed locus $\mathscr{M}^{\mathbb{T}}$.

Let us further assume that the fixed locus $\mathscr{M}^{\mathbb{T}}$ consists of isolated points and that the restriction of the obstruction theory to $\mathscr{M}^{\mathbb{T}}$ has no $\mathbb{T}$-fixed part. These assumptions will hold for the DT moduli space by [MNOP06, Section 4.5]. Under these assumptions, the formula reads

$$
\chi\left(\mathscr{M}, \mathcal{F} \otimes \mathcal{O}^{\text {vir }}\right)=\sum_{p \in \mathscr{M}^{\mathbb{T}}} \chi\left(p,\left.\mathcal{F}\right|_{p}\right) \otimes \operatorname{Sym}^{\bullet}\left(\chi\left(p,\left(\mathcal{T}_{p}^{\text {vir }}\right)^{\vee}\right)\right),
$$

where Sym is as defined in (2.2). By assumption, none of the monomials appearing in the character of any $\mathcal{T}_{p}^{\text {vir }}$ are trivial. So, the operation Sym $\bullet^{\bullet}$ is well defined.

In our applications in Section 6, we also use the more classical variant [Tho92, Theorem 3.5] of K-theoretic equivariant localization where $\mathscr{M}$ is a smooth scheme; in this case, the ordinary structure sheaves and tangent bundles replace their virtual analogs.

Let us now specialize to the situation when $\mathscr{M}$ is a component of $\mathrm{DT}(X)$, the torus $\mathbb{T}$ is the double cover $\tilde{T}$ of $T=\operatorname{diag}\left(t_{1}, t_{2}, t_{3}\right)$, and $\mathcal{F}$ is $\left(\mathcal{K}^{\text {vir }}\right)^{1 / 2}$. The symmetry of the perfect obstruction theory of $\mathrm{DT}(X)$ ensures that the resulting localization expression enjoys a particularly nice form. Given $\mathcal{I} \in \mathrm{DT}(X)^{\tilde{T}}$, we may write the $\tilde{T}$-character of $\mathcal{T}_{\mathcal{I}}^{\text {vir }}$ as

$$
T_{\mathcal{I}}^{\text {vir }}=\operatorname{Def}_{\mathcal{I}}-\operatorname{Obs}_{\mathcal{I}}=\sum_{i} u_{i}-v_{i}
$$

where each $u_{i}$ and $v_{i}$ is a $\tilde{T}$-weight; we also have

$$
\left(\mathcal{K}^{\text {vir }}\right)_{\mathcal{I}}^{1 / 2}=\prod_{i} \frac{v_{i}^{1 / 2}}{u_{i}^{1 / 2}} .
$$

By (2.1), we may reorder the $u_{i}$ and $v_{i}$ so that $v_{i}=\kappa / u_{i}$. By virtual localization, we have the following equality of $\tilde{T}$-characters:

$$
\begin{aligned}
& Z_{\mathrm{DT}}(X)=\sum_{\substack{\beta \in H_{2}(X, \mathbb{Z})^{\mathrm{eff}} \\
n \in \mathbb{Z}}} Q^{n} u^{\beta} \chi\left(\mathrm{DT}(X, \beta, n)^{T},\left.\left(\left(\mathcal{K}^{\mathrm{vir}}\right)^{1 / 2} \otimes \mathrm{Sym}^{\bullet} \mathcal{T}^{\mathrm{vir}}\right)\right|_{\mathrm{DT}(X, \beta, n)^{T}}\right) \\
& =\sum_{\substack{\beta \in H_{2}(X, \mathbb{Z})^{\mathrm{eff}} \\
n \in \mathbb{Z}}} Q^{n} u^{\beta} \sum_{\mathcal{I} \in \mathrm{DT}(X, \beta, n)^{T}} \prod_{i} \frac{\left(\kappa / u_{i}\right)^{1 / 2}-\left(\kappa / u_{i}\right)^{-1 / 2}}{u_{i}^{1 / 2}-u_{i}^{-1 / 2}} .
\end{aligned}
$$

Motivated by this expression, we adopt the following notation from [Oko17, Oko19]. Given 


\section{N. Arbesfeld}

a $T$-character $V=\sum_{i} u_{i}-\sum_{j} v_{j}$, where $u_{i}, v_{j} \in T^{\vee}$, we set

$$
\hat{a}(V)=\frac{\prod_{j} v_{j}^{1 / 2}-v_{j}^{-1 / 2}}{\prod_{i} u_{i}^{1 / 2}-u_{i}^{-1 / 2}} \in \mathbb{Q}\left(t_{1}, t_{2}, t_{3},\left(t_{1} t_{2} t_{3}\right)^{1 / 2}\right) .
$$

\subsection{Independence of the choice of $\left(\mathcal{K}^{\text {vir }}\right)^{1 / 2}$}

If $\mathcal{L}$ is a $\tilde{T}$-equivariant line bundle on $\operatorname{DT}(X)$ with $\mathcal{L}^{\otimes 2} \cong \mathcal{K}^{\text {vir }}$ and $\mathcal{I} \in \operatorname{DT}(X)$ is a torus-fixed sheaf, then the $\tilde{T}$-weight of the fiber $\left.\mathcal{K}^{\text {vir }}\right|_{\mathcal{I}}$ is the square of the $\tilde{T}$-weight of the fiber $\left.\mathcal{L}\right|_{\mathcal{I}}$. It follows from (2.4) that $Z_{\mathrm{DT}}(X)$ does not depend on the choice of square root $\left(\mathcal{K}^{\mathrm{vir}}\right)^{1 / 2}$. This is a special instance of [Tho20, Proposition 2.6].

As any two square roots of $\mathcal{K}^{\text {vir }}$ differ by two-torsion in $\operatorname{Pic}(\operatorname{DT}(X))$, the independence of $Z_{\mathrm{DT}}(X)$ on the choice of square root can also be deduced from equivariant localization and virtual Hirzebruch-Riemann-Roch [FG10, Corollary 3.4].

\section{Slope independence}

For this section, we let $\mathscr{M}$ denote a quasi-projective scheme equipped with an action of a torus $\mathbb{T}$ and a $\mathbb{T}$-equivariant perfect obstruction theory. Examples include smooth schemes, DT moduli space and the Pandharipande-Thomas stable pair moduli space of a threefold (see [PT09]). We assume that $\mathscr{M}^{\mathbb{T}}$ is proper and nonempty. Let $\mathcal{F}$ denote a $\mathbb{T}$-equivariant K-theory class of coherent sheaves on $\mathscr{M}$.

One assumption used in the analysis of K-theoretic enumerative invariants of Calabi-Yau threefolds in [NO16, Section 7] is that the underlying moduli space has proper components. If $\mathscr{M}$ is proper, then the $\mathbb{T}$-character $\chi\left(\mathscr{M}, \mathcal{F} \otimes \mathcal{O}^{\text {vir }}\right)$ is a Laurent polynomial in $\mathbb{T}$. Let $w \in \mathbb{T}^{\vee}$ be a primitive weight. It follows that if the limits $\chi\left(\mathscr{M}, \mathcal{F} \otimes \mathcal{O}^{\text {vir }}\right)^{\sigma}$ exist for generic one-parameter subgroups $\sigma: \mathbb{C}^{\times} \rightarrow \operatorname{ker}(w)$, then $\chi\left(\mathscr{M}, \mathcal{F} \otimes \mathcal{O}^{\text {vir }}\right)$ is a function of $w$ and, moreover, that

$$
\chi\left(\mathscr{M}, \mathcal{F} \otimes \mathcal{O}^{\text {vir }}\right)=\chi\left(\mathscr{M}, \mathcal{F} \otimes \mathcal{O}^{\text {vir }}\right)^{\sigma} \in \mathbb{Q}[w]
$$

for any generic $\sigma$.

In this section, we formulate Proposition 3.4, a statement in the spirit of (3.1) that holds for nonproper moduli spaces $\mathscr{M}$.

\subsection{Denominators in localization}

Given a weight $w \in \mathbb{T}^{\vee}$, we let $\mathbb{T}_{w} \subset \mathbb{T}$ denote the maximal torus inside the subgroup $\operatorname{ker}(w)$ of $\mathbb{T}$.

Definition 3.1. A weight $w \in \mathbb{T}^{\vee}$ is said to be a compact weight of $\mathscr{M}$ if the fixed locus $\mathscr{M}^{\mathbb{T}} w$ is proper, and noncompact otherwise.

While many possible denominators can occur in any particular term of a localization expression for $\chi\left(\mathscr{M}, \mathcal{F} \otimes \mathcal{O}^{\text {vir }}\right)$, the following result restricts the possible denominators that may appear in the total sum.

Proposition 3.2. The $\mathbb{T}$-character $\chi\left(\mathscr{M}, \mathcal{F} \otimes \mathcal{O}^{\text {vir }}\right)$ can be written as a quotient of two Laurent polynomials $p(t) / q(t)$ such that $q(t)$ is of the form $\prod(1-w)$, where each $w$ is a noncompact weight of $\mathscr{M}$. 


\section{K-THEORETiC DT theORY AND Hilbert SCHEMES}

Proof. Write $\chi\left(\mathscr{M}, \mathcal{F} \otimes \mathcal{O}^{\text {vir }}\right)$ as a quotient $f(t)=p(t) / q(t)$, where $q(t)=\prod(1-w)$ and each $w$ is a weight in $\mathcal{N}_{\mathscr{M} / \mathscr{M}^{\mathbb{T}}}^{\mathrm{vir}}$; if $p(t)$ is zero, there is nothing to show.

If $w$ is a compact weight, then equivariant localization with respect to $\mathbb{T}_{w}$ implies that as $\mathbb{T}_{w}$-characters, we have

$$
\chi\left(\mathscr{M}, \mathcal{F} \otimes \mathcal{O}^{\text {vir }}\right) \in \mathbb{Q}\left[t_{i}^{ \pm}, \frac{1}{1-v}\right],
$$

where $v$ ranges over the $\mathbb{T}$-weights occurring in $\mathcal{N}_{\mathscr{M} / \mathscr{M}^{\mathbb{T}} w}^{\mathrm{vir}}$. No such $v$ vanishes on $\mathbb{T}_{w}$, so we conclude that $f(t)$ may be written in a form whose denominator is a product of terms of the form $(1-v)$ where no $v$ is a power of $w$. In particular, the rational function $f(t)$ has no poles at $1=\zeta w$ for any root of unity $\zeta$.

For example, consider the Hilbert scheme $\mathrm{DT}\left(\mathbb{C}^{3}, 0, n\right)$ of $n$ points on $\mathbb{C}^{3}$ with the standard action of a three-dimensional torus $\operatorname{diag}\left(t_{1}, t_{2}, t_{3}\right)$. Then the fixed locus

$$
\operatorname{DT}\left(\mathbb{C}^{3}, 0, n\right)^{\operatorname{diag}\left(t_{1}, t_{2}, t_{3}\right)_{w}}
$$

is proper unless $w$ is a nontrivial power of some $t_{k}$, so the only noncompact directions are of the form $t_{k}^{i}$. Nekrasov's formula [Oko17, Theorem 3.3.6] furnishes an explicit formula for the K-theoretic DT partition function:

$$
\sum_{n=0}^{\infty}(-Q)^{n} \chi\left(\mathrm{DT}\left(\mathbb{C}^{3}, 0, n\right), \tilde{\mathcal{O}}^{\text {vir }}\right)=\operatorname{Sym} \bullet\left(\frac{-Q}{\left(1-Q \kappa^{1 / 2}\right)\left(1-Q \kappa^{-1 / 2}\right)} \prod_{k=1}^{3} \frac{\kappa^{1 / 2} t_{k}^{-1}-\kappa^{-1 / 2}}{1-t_{k}^{-1}}\right)
$$

(where the right-hand side is defined by extending (2.3) in a straightforward manner). In particular, note that any $\chi\left(\mathrm{DT}\left(\mathbb{C}^{3}, 0, n\right), \tilde{\mathcal{O}}^{\text {vir }}\right)$ can be written as a Laurent polynomial whose denominator is a product of terms of the form $\left(1-t_{k}^{m}\right)$.

\subsection{Limit independence}

Let $A \subset \mathbb{T}$ be a subtorus, and let $f(t) \in \mathbb{C}\left(t_{k}\right)$ be a rational function that can be written in the form

$$
\frac{p(t)}{\prod_{i}\left(1-w_{i}\right)},
$$

where $p(t)$ is a Laurent polynomial in $t_{k}$ and the $w_{i} \in \mathbb{T}^{\vee}$ are nontrivial weights. Set $W=\left\{w_{i}\right\}$. Via the map $\mathbb{T}^{\vee} \rightarrow A^{\vee}$, the elements of $W$ can be regarded as $A$-weights.

We say that the rational function $f(t)$ is $A$-balanced if

$$
\lim _{z \rightarrow 0} f(\sigma(z) t)
$$

exists for all one-parameter subgroups $\sigma: \mathbb{C}^{\times} \rightarrow A$ or, equivalently (for nonzero $f$ ), if the set of $A$-weights appearing in the numerator of $f$ is the same as the set of $A$-weights appearing in the denominator of $f$.

Given an $A$-balanced rational function $f$, the weights in $W$ impose a wall and chamber structure on the lattice of one-parameter subgroups $\mathbb{C}^{\times} \rightarrow A$. We show that the limit of $f$ under a one-parameter subgroup $\sigma: \mathbb{C}^{\times} \rightarrow A$ depends only on the chamber containing $\sigma$.

We restrict our focus to one-parameter subgroups lying in the interior of such a chamber: we say that a one-parameter subgroup $\sigma: \mathbb{C}^{\times} \rightarrow A$ is $f$-generic if, for all $w_{i} \in W$, one has 


\section{N. Arbesfeld}

$w_{i}(\sigma(z) t) \neq w_{i}(t)$. Given an $f$-generic $\sigma$ and $w_{i} \in W$, we have $w_{i}(\sigma(z) t)=z^{r} w_{i}(t)$ for some $r \neq 0$. Call $w$ attracting with respect to $\sigma$ if $r>0$ and repelling with respect to $\sigma$ if $r<0$.

Proposition 3.3. Let

$$
f(t)=\frac{p(t)}{\prod_{i}\left(1-w_{i}\right)}
$$

be $A$-balanced, and let $\sigma_{1}$ and $\sigma_{2}$ be two $f$-generic one-parameter subgroups sharing the same attracting or repelling behavior for each weight $w_{i}$. Then

$$
\lim _{z \rightarrow 0} f\left(\sigma_{1}(z) t\right)=\lim _{z \rightarrow 0} f\left(\sigma_{2}(z) t\right) .
$$

Proof. Multiplying the numerator and denominator of $f$ by a Laurent monomial if necessary, we may assume that each $w_{i}$ is attracting with respect to both $\sigma_{1}$ and $\sigma_{2}$. Set $q(t)=\prod_{i}\left(1-w_{i}\right)$; then we may write $q\left(\sigma_{1}(z) t\right)=1+z q_{1}(t, z)$, where $q_{1} \in \mathbb{C}\left[t_{k}^{ \pm}, z\right]$.

Via the map $\varphi: \mathbb{T}^{\vee} \rightarrow A^{\vee}$, we regard the monomials appearing in the numerator and denominator of $f$ as $A$-weights. Write $p(t)=\sum c_{j} u_{j}$, where each $u_{j}$ is a monomial, and set

$$
p_{0}(t)=\left\{\sum c_{j} u_{j} \mid \varphi\left(u_{j}\right)=1 \in A^{\vee}\right\} .
$$

The set of $A$-weights appearing in the numerator of $f$ is the same as the set of $A$-weights appearing in the denominator of $f$. Therefore, we may write

$$
p\left(\sigma_{1}(z) t\right)=p_{0}(t)+z p_{1}(t, z),
$$

where $p_{1} \in \mathbb{C}\left[t_{k}^{ \pm}, z\right]$ and $p_{0}$ is regarded as an element of $\mathbb{C}\left[t_{k}^{ \pm}, z\right]$ via the inclusion $\mathbb{C}\left[t_{k}^{ \pm}\right] \hookrightarrow$ $\mathbb{C}\left[t_{k}^{ \pm}, z\right]$. It follows that

$$
\lim _{z \rightarrow 0} f\left(\sigma_{1}(z) t\right)=\frac{p_{0}(t)+z p_{1}(t, z)}{1+z q_{1}(t)}=p_{0}(t)
$$

the same is true of $\sigma_{2}$.

\subsection{Limit independence for Euler characteristics}

Again, let $A \subset \mathbb{T}$ be a subtorus. We say that a one-parameter subgroup $\sigma: \mathbb{C}^{\times} \rightarrow A$ is $\mathscr{M}$-generic if for any noncompact weight $w$ of $\mathscr{M}$, we have $w(\sigma(z) t) \neq w(t)$. Combining Propositions 3.2 and 3.3 , we obtain the following.

Proposition 3.4. If $\chi\left(\mathscr{M}, \mathcal{F} \otimes \mathcal{O}^{\text {vir }}\right)$ is $A$-balanced and $\sigma_{1}$ and $\sigma_{2}$ are two $\mathscr{M}$-generic oneparameter subgroups $\mathbb{C}^{\times} \rightarrow A$ with the same attracting or repelling behavior for each noncompact weight of $\mathscr{M}$, then $\chi\left(\mathscr{M}, \mathcal{F} \otimes \mathcal{O}^{\text {vir }}\right)^{\sigma_{1}}=\chi\left(\mathscr{M}, \mathcal{F} \otimes \mathcal{O}^{\text {vir }}\right)^{\sigma_{2}}$.

Now, specialize $\mathscr{M}$ to be a component of DT moduli space, the torus $\mathbb{T}$ to be the double cover $\tilde{T}$ of the torus $T=\operatorname{diag}\left(t_{1}, t_{2}, t_{3}\right)$ as in the previous section, the sheaf $\mathcal{F}$ to be $\left(\mathcal{K}^{\text {vir }}\right)^{1 / 2}$ and $A$ to be the Calabi-Yau torus $\operatorname{ker}(\kappa)$, where $\kappa$ is the character of $\left(\mathcal{K}^{\mathrm{vir}}\right)^{\vee}$. By $(2.4)$, the character $\chi\left(\operatorname{DT}(X, \beta, n), \tilde{\mathcal{O}}^{\text {vir }}\right)$ is $A$-balanced. We obtain Theorem 1.5.

\section{Preferred limits of the DT partition function}

Let $X$ be a smooth toric Calabi-Yau threefold, and let $T$ be the torus $\operatorname{diag}\left(t_{1}, t_{2}, t_{3}\right)$ acting on $X$. Choose this action such that the anticanonical bundle $\mathcal{K}_{X}^{\vee}$ is scaled with weight $\kappa=t_{1} t_{2} t_{3}$. 


\section{K-THEORETIC DT THEORY AND HilBerT SCHEMES}

\subsection{K-theoretic building blocks}

We recall from [MNOP06, NO16, Oko19] a procedure for writing the partition functions $Z_{\mathrm{DT}}(X)$ and $Z_{\mathrm{DT}}^{\prime}(X)$ in terms of combinatorial expressions associated with the vertices and edges of the toric diagram $\Delta(X)$.

4.1.1 Torus-fixed locus. The fixed locus DT $(X)^{T}$ consists of isolated points, with finitely many in any component $\mathrm{DT}(X, \beta, n)$. We recall from [MNOP06, Sections 4.1-4.2] a combinatorial description of this fixed-point locus.

The points of $X^{T}$ correspond to the vertices of the toric polytope $\Delta(X)$, and the $T$-invariant rational curves of $X$ correspond to the bounded edges of $\Delta(X)$. For $x_{i} \in X^{T}$, let $U_{i}$ be the toric chart centered at $x_{i}$. Suppose that $x_{i}, x_{j} \in X^{T}$ are connected by a $T$-invariant rational curve $C_{i j} \subset X$; then $U_{i} \cap U_{j}$ is the total space of the direct sum of two line bundles $\mathcal{O}\left(l_{i j}\right) \oplus \mathcal{O}\left(l_{i j}^{\prime}\right)$ over $C_{i j}$.

Choose coordinates on each $U_{i}$ such that the torus $T$ scales the coordinate directions by $T$ weights $w_{i_{1}}, w_{i_{2}}, w_{i_{3}}$; after an orientation on the one-skeleton of $\Delta(X)$ is fixed, these coordinates can be compatibly ordered at each fixed point. The edges in the toric polytope emanating from the fixed point $x_{i}$ correspond to coordinate axes. Let $z_{i_{1}}, z_{i_{2}}, z_{i_{3}}$ denote the corresponding coordinate functions on $U_{i}$ so that $U_{i}=\operatorname{Spec} \mathbb{C}\left[z_{i_{1}}, z_{i_{2}}, z_{i_{3}}\right]$. The torus $T$ scales these coordinate functions by $w_{i_{1}}^{-1}, w_{i_{2}}^{-1}, w_{i_{3}}^{-1}$, respectively. After cyclically permuting coordinates and exchanging $l_{i j}$ with $l_{i j}^{\prime}$, if necessary, the weights of coordinate directions on $U_{i}$ and $U_{j}$ are identified by

$$
w_{j_{1}}=w_{i_{1}}^{-1}, \quad w_{j_{2}}=w_{i_{1}}^{-l_{i j}^{\prime}} w_{i_{3}}, \quad w_{j_{3}}=w_{i_{1}}^{-l_{i j}} w_{i_{2}} ;
$$

see Figure 4. The convention is chosen so that the first coordinate corresponds to the edge of the polytope connecting the two vertices, and the order of the remaining coordinates is determined by the orientation on $\Delta(X)$.

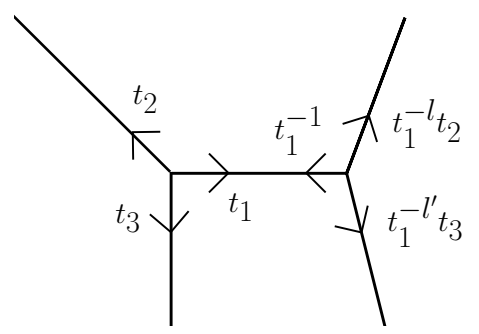

Figure 4: The weights along a $T$-invariant rational curve with normal bundle $\mathcal{O}(l) \oplus \mathcal{O}\left(l^{\prime}\right)$

A $T$-fixed ideal sheaf $\mathcal{I} \in \mathrm{DT}(X)$ is determined by a collection of $T$-fixed ideals $\left\{\mathcal{I}_{i} \subset \mathcal{O}_{U_{i}}\right\}$ whose restrictions $\left.\mathcal{I}_{i}\right|_{U_{i} \cap U_{j}}$ are compatible and glue to an ideal sheaf that cuts out a proper subscheme of $X$.

A $T$-fixed ideal $\mathcal{I}_{i} \subset U_{i}$ is given by a monomial ideal $I_{i} \subset \mathbb{C}\left[z_{i_{1}}, z_{i_{2}}, z_{i_{3}}\right]$. There is a one-to-one correspondence between monomial ideals and three-dimensional partitions $\pi_{i}$ given by

$$
I_{i} \leftrightarrow \pi_{i}=\left\{\left(b_{1}, b_{2}, b_{3}\right) \mid z_{i_{1}}^{b_{1}} z_{i_{2}}^{b_{2}} z_{i_{3}}^{b_{3}} \notin I_{i}\right\} ;
$$

we pass freely between an ideal and its associated partition.

The condition that $\mathcal{I}$ cuts out a proper subscheme restricts the possible asymptotics of the 


\section{N. Arbesfeld}

three-dimensional partitions $\pi_{i}$. The asymptotics of each such $\pi_{i}$ along each coordinate direction must be finite (as described in Section 1.4); more restrictively, the partition $\pi_{i}$ at a given fixed point $x_{i}$ must satisfy the following condition:

$$
\pi_{i}^{(k)} \neq \varnothing \text { only if the edge of } \Delta(X) \text { in the direction of } z_{i_{k}} \text { is bounded. }
$$

The asymptotics of the partitions must also agree in the direction of any $T$-invariant rational curve. To be precise, given $x_{i}, x_{j} \in X^{T}$ connected by a $T$-invariant rational curve $C_{i j}$, the restrictions $\left.\mathcal{I}_{i}\right|_{U_{i} \cap U_{j}}$ and $\left.\mathcal{I}_{j}\right|_{U_{i} \cap U_{j}}$ are compatible if the two localizations

$$
\left(I_{i}\right)_{z_{i_{1}}} \subset \mathbb{C}\left[z_{i_{1}}^{ \pm 1}, z_{i_{2}}, z_{i_{3}}\right], \quad\left(I_{j}\right)_{z_{i_{1}}^{-1}} \subset \mathbb{C}\left[z_{i_{1}}^{ \pm 1}, z_{i_{2}}, z_{i_{3}}\right]
$$

coincide. In terms of three-dimensional partitions, this compatibility translates to the condition that

$$
\pi_{i}^{(1)}=\left(\pi_{j}^{(1)}\right)^{t}
$$

see Figure 5.
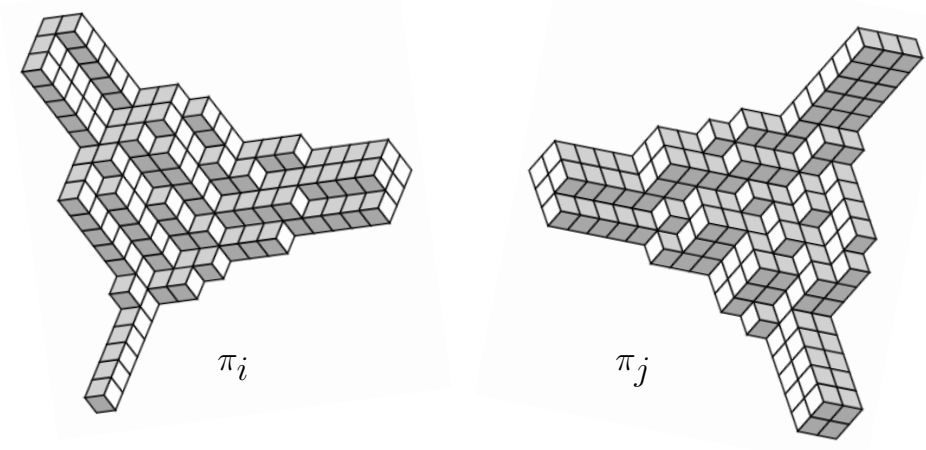

Figure 5: Two compatible partitions $\pi_{i}$ and $\pi_{j}$ at vertices connected by an edge of $\Delta(X)$

Conversely, any collection of three-dimensional partitions $\pi_{i}$ satisfying (4.2) and (4.3) corresponds to a $T$-fixed ideal sheaf $\mathcal{I}$. It follows that a point of $\operatorname{DT}(X)^{T}$ is specified by a collection $\left\{\lambda_{i j}, \pi_{i}\right\}$ consisting of, first, a choice of two-dimensional partition $\lambda_{i j}$ for each bounded edge of $\Delta(X)$ and, then, a choice of three-dimensional partition $\pi_{i}$ for each vertex of $\Delta(X)$ whose asymptotics coincide with the chosen $\lambda_{i j}$. By convention, we set

$$
\lambda_{j i}=\lambda_{i j}^{t} .
$$

4.1.2 Obstruction theory at fixed points. Recall from [MNOP06, Section 4.6] that the virtual tangent space to $\mathrm{DT}(X)$ at an ideal sheaf $\mathcal{I}$ is given by

$$
\mathcal{T}_{\mathcal{I}}^{\text {vir }}=\operatorname{Ext}^{1}(\mathcal{I}, \mathcal{I})-\operatorname{Ext}^{2}(\mathcal{I}, \mathcal{I})
$$

Given a torus character $V$, let $V^{\vee}$ denote the character of the dual representation.

As $X$ is Calabi-Yau, the obstruction theory on $\mathrm{DT}(X)$ is symmetric. So, as characters,

$$
T_{\mathcal{I}}^{\mathrm{vir}}=-\left(T_{\mathcal{I}}^{\mathrm{vir}}\right)^{\vee} \cdot \kappa
$$




\section{K-THEORETIC DT THEORY AND HilBerT SCHEMES}

The obstruction theory at a torus-fixed point $\mathcal{I} \in \mathrm{DT}(X)^{T}$ can be calculated from the corresponding combinatorial data $\left\{\lambda_{i j}, \pi_{i}\right\}$. We recall this procedure from [MNOP06, Sections 4.7-4.9]; see also [NO16, Section 8.2] and [Oko19, Section 6.3].

Now suppose that $T=\operatorname{diag}\left(t_{1}, t_{2}, t_{3}\right)$ acts on $\mathbb{C}^{3}=\operatorname{Spec} \mathbb{C}\left[z_{1}, z_{2}, z_{3}\right]$ so that $T$ scales the coordinate $z_{k}$ by $t_{k}^{-1}$. Given a partition $\pi$ corresponding to a $T$-fixed ideal sheaf $\mathcal{I} \subset \mathcal{O}_{\mathbb{C}^{3}}$, we set $O_{\pi}$ to be the $T$-character $\chi\left(\mathcal{O}_{\mathbb{C}^{3}} / \mathcal{I}\right)$, that is,

$$
O_{\pi}=\sum_{\square \in \pi} t_{1}^{-b_{1}} t_{2}^{-b_{2}} t_{3}^{-b_{3}}
$$

For $\pi$ with finitely many boxes, we have

$$
T_{\pi}^{\mathrm{vir}}\left(t_{1}, t_{2}, t_{3}\right)=O_{\pi}-O_{\pi}^{\vee} t_{1} t_{2} t_{3}-O_{\pi} O_{\pi}^{\vee}\left(1-t_{1}\right)\left(1-t_{2}\right)\left(1-t_{3}\right) ;
$$

this is a Laurent polynomial in the variables $t_{k}$. For $\pi$ with infinitely many boxes, we first write the infinite sum $O_{\pi}$ as a rational function and define the tangent space $T_{\pi}^{\mathrm{vir}}$ to be the rational function in $t_{k}$ given by the same formula (4.5).

For a $T$-fixed ideal sheaf $\mathcal{I} \in \mathrm{DT}(X)^{T}$ given by $\left\{\lambda_{i j}, \pi_{i}\right\}$ with weights $w_{i_{1}}, w_{i_{2}}, w_{i_{3}}$ as above, equivariant localization implies

$$
T_{\mathcal{I}}^{\mathrm{vir}}=\sum_{i} T_{\pi_{i}}^{\mathrm{vir}}\left(w_{i_{1}}, w_{i_{2}}, w_{i_{3}}\right)
$$

While each term on the right-hand side of (4.6) is a rational function, the total sum is a Laurent polynomial in $t_{k}$. It is explained in [MNOP06, Section 4.9] (see also [NO16, Sections 8.2.28.2.3], [Oko19, Section 6.3.1]) how to write $T_{\mathcal{I}}^{\text {vir }}$ as a sum of Laurent polynomials by regularizing each term of (4.6) by contributions associated with the asymptotics of the corresponding partition $\pi_{i}$. Recalling (1.6), set

$$
T_{\lambda_{i j}}\left(w_{i_{k+1}}, w_{i_{k+2}}\right)=\sum_{\square \in \lambda_{i j}} w_{i_{k+1}}^{-l(\square)} w_{i_{k+2}}^{a(\square)+1}+w_{i_{k+1}}^{l(\square)+1} w_{i_{k+2}}^{-a(\square)} .
$$

Given a $T$-invariant rational curve $C_{i j}$ corresponding to coordinate direction $z_{i_{k}}$, define

$$
E_{\lambda_{i j},\left(l_{i j}, l_{i j}^{\prime}\right)}\left(w_{i_{1}}, w_{i_{2}}, w_{i_{3}}\right)=\frac{T_{\lambda_{i j}}\left(w_{i_{k+1}}, w_{i_{k+2}}\right)}{1-w_{i_{k}}^{-1}}+\frac{T_{\lambda_{i j}^{t}}\left(w_{i_{k}}^{-l_{i j}^{\prime}} \cdot w_{i_{k+2}}, w_{i_{k}}^{-l_{i j}} \cdot w_{i_{k+1}}\right)}{1-w_{i_{k}}} ;
$$

here, for $k=1,2,3$, set $w_{i_{k+3}}=w_{i_{k}}$. One can use (4.7) to write (4.8) in terms of arm and leg lengths. We omit $\left(l_{i j}, l_{i j}^{\prime}\right)$ from the notation for $E$ when the meaning is clear.

We then define

$$
V_{\pi_{i}}\left(w_{i_{1}}, w_{i_{2}}, w_{i_{3}}\right)=T_{\pi_{i}}^{\mathrm{vir}}\left(w_{i_{1}}, w_{i_{2}}, w_{i_{3}}\right)-\sum_{k=1}^{3} \frac{T_{\pi_{i}^{(k)}}\left(w_{i_{k+1}}, w_{i_{k+2}}\right)}{1-w_{i_{k}}^{-1}} .
$$

By [MNOP06, Lemma 9], each $V_{\pi_{i}}$ and $E_{\lambda_{i j}}$ is a Laurent polynomial in $t_{i}$. From (4.6), we have

$$
T_{\mathcal{I}}^{\mathrm{vir}}=\sum_{i} V_{\pi_{i}}\left(w_{i_{1}}, w_{i_{2}}, w_{i_{3}}\right)+\sum_{i j} E_{\lambda_{i j},\left(l_{i j}, l_{i j}^{\prime}\right)}\left(w_{i_{1}}, w_{i_{2}}, w_{i_{3}}\right) .
$$

It follows from (4.5), (4.8), (4.9) that each term of (4.10) enjoys the symmetry (4.4):

$$
V_{\pi_{i}}\left(t_{1}, t_{2}, t_{3}\right)=-V_{\pi_{i}}\left(t_{1}, t_{2}, t_{3}\right)^{\vee} \cdot \kappa, \quad E_{\lambda_{i j}}\left(t_{1}, t_{2}, t_{3}\right)=-E_{\lambda_{i j}}\left(t_{1}, t_{2}, t_{3}\right)^{\vee} \cdot \kappa .
$$




\section{N. Arbesfeld}

4.1.3 Euler characteristic. The value of $\chi\left(\mathcal{O}_{Y}\right)$ for a $T$-fixed $Y \in \mathrm{DT}(X)$ can also be computed in terms of edge and regularized vertex contributions using the Čech cover $\left\{U_{i}\right\}$. We recall the description from [MNOP06, Lemma 5].

Set

$$
\chi(\pi)=\sum_{\square \in \pi}(1-(\text { number of legs }\{\pi\langle 1\rangle, \pi\langle 2\rangle, \pi\langle 3\rangle\} \text { that contain } \square)) ;
$$

this is the renormalized volume of $\pi$ as defined in [MNOP06, Section 4.4]. Only finitely many boxes of $\pi$ contribute to the sum (4.12).

For a two-dimensional partition $\lambda$ and integers $\left(l, l^{\prime}\right)$, set

$$
\chi\left(\lambda,\left(l, l^{\prime}\right)\right)=\sum_{\square \in \lambda} 1-l b_{1}-l^{\prime} b_{2} .
$$

Then, for $\mathcal{I} \in \mathrm{DT}(X)^{T}$ specified by partition data $\left\{\lambda_{i j}, \pi_{i}\right\}$, we have

$$
\chi(\mathcal{O} / \mathcal{I})=\sum_{i} \chi\left(\pi_{i}\right)+\sum_{i, j} \chi\left(\lambda_{i j},\left(l_{i j}, l_{i j}^{\prime}\right)\right) .
$$

\subsection{Factorization of components of the partition function}

We rewrite (2.4) as

$$
Z_{\mathrm{DT}}(X)=\sum_{\mathcal{I} \in \mathrm{DT}(X)^{T}} Q^{\chi(\mathcal{O} / \mathcal{I})} u^{[\mathcal{O} / \mathcal{I}]} \hat{a}\left(T_{\mathcal{I}}^{\mathrm{vir}}\right)
$$

We now write $Z_{\mathrm{DT}}(X)$ in terms of expressions associated with the vertices and edges of $\Delta(X)$. Let $\mathcal{I} \in \mathrm{DT}(X)^{T}$ be given by the configuration $\left\{\lambda_{i j}, \pi_{i}\right\}$. Then, by (4.10),

$$
\hat{a}\left(T_{\mathcal{I}}^{\mathrm{vir}}\right)=\prod_{i} \hat{a}\left(V_{\pi_{i}}\left(w_{i_{1}}, w_{i_{2}}, w_{i_{3}}\right)\right) \prod_{i j} \hat{a}\left(E_{\lambda_{i j},\left(l_{i j}, l_{i j}^{\prime}\right)}\left(w_{i_{1}}, w_{i_{2}}, w_{i_{3}}\right)\right),
$$

while

$$
Q^{\chi(\mathcal{O} / \mathcal{I})}=\prod_{i} Q^{\chi\left(\pi_{i}\right)} \prod_{i j} Q^{\chi\left(\lambda_{i j},\left(l_{i j}, l_{i j}^{\prime}\right)\right)}, \quad u^{[\mathcal{O} / \mathcal{I}]}=\prod_{i j} u^{\left|\lambda_{i j}\right|\left[C_{i j}\right]} .
$$

Given two-dimensional partitions $\lambda_{1}, \lambda_{2}, \lambda_{3}$, set $P\left(\lambda_{1}, \lambda_{2}, \lambda_{3}\right)$ to be the set of three-dimensional partitions $\pi$ whose asymptotics are given by $\lambda_{1}, \lambda_{2}, \lambda_{3}$, that is,

$$
P\left(\lambda_{1}, \lambda_{2}, \lambda_{3}\right)=\left\{\pi \text { a three-dimensional partition } \mid \pi^{(k)}=\lambda_{k}, k \in\{1,2,3\}\right\} .
$$

Then, set

$$
\hat{E}\left(\lambda,\left(l, l^{\prime}\right)\right)\left(w_{i_{1}}, w_{i_{2}}, w_{i_{3}}\right)=Q^{\chi\left(\lambda,\left(l, l^{\prime}\right)\right)} \hat{a}\left(E_{\lambda,\left(l, l^{\prime}\right)}\left(w_{i_{1}}, w_{i_{2}}, w_{i_{3}}\right)\right)
$$

and

$$
\hat{V}(\lambda, \mu, \nu)\left(w_{i_{1}}, w_{i_{2}}, w_{i_{3}}\right)=\sum_{\pi \in P(\lambda, \mu, \nu)} Q^{\chi(\pi)} \hat{a}\left(V_{\pi}\left(w_{i_{1}}, w_{i_{2}}, w_{i_{3}}\right)\right) .
$$

We may then write $Z_{\mathrm{DT}}(X)$ as

$$
\sum_{\left\{\lambda_{i j}\right\}}\left(\prod_{i j} u^{\left|\lambda_{i j}\right|\left[C_{i j}\right]} \hat{E}\left(\lambda_{i j},\left(l_{i j}, l_{i j}^{\prime}\right)\right)\left(w_{i_{1}}, w_{i_{2}}, w_{i_{3}}\right) \prod_{i} \hat{V}\left(\lambda_{i 1}, \lambda_{i 2}, \lambda_{i 3}\right)\left(w_{i_{1}}, w_{i_{2}}, w_{i_{3}}\right)\right),
$$

where the sum is over all assignments of two-dimensional partitions to the bounded edges of $\Delta(X)$. The expression $\hat{V}(\lambda, \mu, \nu)\left(t_{1}, t_{2}, t_{3}\right)$ is called the $K$-theoretic equivariant vertex. 


\section{K-THEORETIC DT THEORY AND HilBerT SCHEMES}

4.2.1 Reduced partition function. The reduced K-theoretic partition function admits a similar expression. From (4.16), we see that we may write $Z_{\mathrm{DT}}^{\prime}(X)$ as

$$
\sum_{\left\{\lambda_{i j}\right\}}\left(\prod_{i j} u^{\left|\lambda_{i j}\right|\left[C_{i j}\right]} \hat{E}\left(\lambda_{i j},\left(l_{i j}, l_{i j}^{\prime}\right)\right)\left(w_{i_{1}}, w_{i_{2}}, w_{i_{3}}\right) \prod_{i} \frac{\hat{V}\left(\lambda_{i 1}, \lambda_{i 2}, \lambda_{i 3}\right)\left(w_{i_{1}}, w_{i_{2}}, w_{i_{3}}\right)}{\hat{V}(\varnothing, \varnothing, \varnothing)\left(w_{i_{1}}, w_{i_{2}}, w_{i_{3}}\right)}\right) .
$$

The expression $\hat{V}(\varnothing, \varnothing, \varnothing)$ is completely characterized by Nekrasov's formula (3.2), so that the difference between $Z_{\mathrm{DT}}(X)$ and $Z_{\mathrm{DT}}^{\prime}(X)$ is an easily understood prefactor. Our formulas will be more concise when formulated in terms of $Z_{\mathrm{DT}}^{\prime}(X)$. Set

$$
\hat{V}^{\prime}\left(\lambda_{i 1}, \lambda_{i 2}, \lambda_{i 3}\right)\left(w_{i_{1}}, w_{i_{2}}, w_{i_{3}}\right)=\frac{\hat{V}\left(\lambda_{i 1}, \lambda_{i 2}, \lambda_{i 3}\right)\left(w_{i_{1}}, w_{i_{2}}, w_{i_{3}}\right)}{\hat{V}(\varnothing, \varnothing, \varnothing)\left(w_{i_{1}}, w_{i_{2}}, w_{i_{3}}\right)} .
$$

\subsection{Preferred limits of vertex and edge contributions}

4.3.1 Rigidity. The symmetry (4.11) enjoyed by the vertex and edge contributions ensures that each contribution behaves well under limits of generic one-parameter subgroups. Let $A \subset T$ be the Calabi Yau subtorus $\operatorname{ker}(\kappa)$. Recall that such one-parameter subgroups $\sigma: \mathbb{C}^{\times} \rightarrow A$ are called slopes.

It is explained in [MNOP06] that no weight appearing in $T_{\mathcal{I}}^{\mathrm{vir}}$ for $\mathcal{I} \in \mathrm{DT}(X)^{T}$ is a power of $\kappa$. Let $w$ be a weight appearing in Def ${ }^{\text {vir }}$ and $\sigma: \mathbb{C}^{\times} \rightarrow A$ be a slope such that $w(\sigma(z) t)=z^{r} w(t)$, where $r \neq 0$. Then, we have

$$
\begin{aligned}
\lim _{z \rightarrow 0} \frac{(\kappa / w(\sigma(z) t))^{1 / 2}-(\kappa / w(\sigma(z) t))^{-1 / 2}}{(w(\sigma(z) t))^{1 / 2}-(w(\sigma(z) t))^{-1 / 2}} & =\lim _{z \rightarrow 0} \frac{z^{-r / 2}(\kappa / w)^{1 / 2}-z^{r / 2}(\kappa / w)^{-1 / 2}}{z^{r / 2} w^{1 / 2}-z^{-r / 2} w^{-1 / 2}} \\
& = \begin{cases}-\kappa^{1 / 2} & \text { if } r>0 \\
-\kappa^{-1 / 2} & \text { if } r<0\end{cases}
\end{aligned}
$$

Given a virtual $T$-character $V$ such that

$$
V=-V^{\vee} \cdot \kappa
$$

we may write

$$
V=\sum_{i} u_{i}-v_{i}
$$

where each $u_{i}$ and $v_{i}$ are $T$-weights and $u_{i} v_{i}=\kappa$. A slope $\sigma: \mathbb{C}^{\times} \rightarrow A$ is said to be $V$-generic if $u_{i}(\sigma(z) t) \neq u_{i}(t)$ for all $u_{i}$; as $u_{i} v_{i}=\kappa$, we also have $v_{i}(\sigma(z) t) \neq v_{i}(t)$.

From (4.14), we see that for any fixed $\beta$, the fixed-point set $\operatorname{DT}(X, \beta, n)^{T}$ is finite for any $n$ and empty for $n \ll 0$. From (4.18), we conclude that for generic $\sigma: \mathbb{C}^{\times} \rightarrow A$,

$$
\sum_{n} Q^{n} \chi\left(\mathrm{DT}(X, \beta, n), \tilde{\mathcal{O}}^{\text {vir }}\right)^{\sigma} \in \Gamma
$$

where $\Gamma \subset \mathbb{Q}\left[\left[Q^{ \pm 1}, \kappa^{ \pm 1 / 2}\right]\right]$ is the integral domain

$$
\left\{\begin{array}{l|l}
\sum_{i, j \in \mathbb{Z}} c_{i, j} Q^{i} \kappa^{j / 2} & \begin{array}{l}
c_{i, j} \in \mathbb{Q}, \\
\text { there exists an } N \text { such that } c_{i, j}=0 \text { whenever } i<N, \\
\text { and for any fixed } i, \text { only finitely many } c_{i, j} \text { are nonzero }
\end{array}
\end{array}\right\} .
$$




\section{N. ARBESfEld}

Definition 4.1 ([NO16, Section 7.3.1]). Given a virtual T-character

$$
V=\sum_{i} u_{i}-v_{i}
$$

with $u_{i} v_{i}=\kappa$ and a $V$-generic slope $\sigma$, define $\operatorname{ind}_{\sigma}(V) \in \mathbb{Z}$ to be the number of weights of $u_{i}$ that are attracting with respect to $\sigma$ (in the sense of Section 3.2) minus the number of $u_{i}$ that are repelling with respect to $\sigma$.

For integers $r_{1}, r_{2}, r_{3}$ with $r_{1}+r_{2}+r_{3}=0$, let $\sigma\left(r_{1}, r_{2}, r_{3}\right): \mathbb{C}^{\times} \rightarrow A$ denote the slope

$$
z \mapsto\left(z^{r_{1}}, z^{r_{2}}, z^{r_{3}}\right) \text {. }
$$

It follows from (4.18) that, for $V$ as in Definition 4.1, we have

$$
(\hat{V})^{\sigma}=\left(-\kappa^{1 / 2}\right)^{\operatorname{ind}_{\sigma}(V)} .
$$

A generic slope $\sigma$ is called a preferred slope if one of the exponents $r_{k}$ has very small magnitude compared to the other two; see the discussion after Corollary 4.5 for a more precise definition.

\subsubsection{Edge limits. Set}

$$
t=-Q \kappa^{1 / 2}, \quad q=-Q \kappa^{-1 / 2}
$$

For the threefolds $X$ considered in Section 5, the possible normal bundles to a $T$-invariant rational curve $C_{i j} \subset X$ are $\mathcal{O}(-1) \oplus \mathcal{O}(-1)$ and $\mathcal{O} \oplus \mathcal{O}(-2)$. We compute the limits of the edge terms $\hat{E}_{\lambda}^{\sigma}$ associated with these two configurations for preferred $\sigma$ as monomials in $q, t, \sqrt{q t}$.

We first consider a $T$-invariant rational curve whose normal bundle in $X$ is $\mathcal{O}(-1) \oplus \mathcal{O}(-1)$, as depicted in Figure 6.

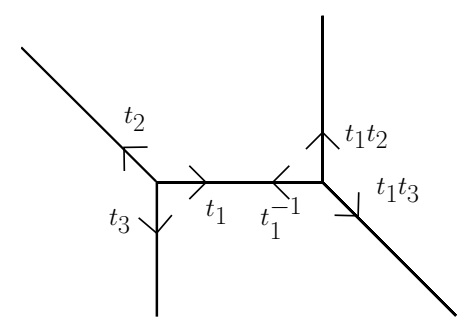

Figure 6: The weights along a $T$-invariant rational curve with normal bundle $\mathcal{O}(-1) \oplus \mathcal{O}(-1)$

It follows from (4.7) that

$$
\begin{aligned}
E_{\lambda,(-1,-1)}\left(t_{1}, t_{2}, t_{3}\right)= & \sum_{\square \in \lambda \mid a(\square)<l(\square)}\left(1+\cdots+t_{1}^{-l(\square)+a(\square)+1}\right) t_{2}^{-l(\square)} t_{3}^{a(\square)+1} \\
& +\sum_{\square \in \lambda \mid a(\square)<l(\square)}-\left(t_{1}+\cdots+t_{1}^{l(\square)-a(\square)}\right) t_{2}^{l(\square)+1} t_{3}^{-a(\square)} \\
& +\sum_{\square \in \lambda \mid a(\square)>l(\square)}-\left(t_{1}+\cdots+t_{1}^{-l(\square)+a(\square)}\right) t_{2}^{-l(\square)} t_{3}^{a(\square)+1} \\
& +\sum_{\square \in \lambda \mid a(\square)>l(\square)}\left(1+\cdots+t_{1}^{l(\square)-a(\square)+1}\right) t_{2}^{l(\square)+1} t_{3}^{-a(\square)} .
\end{aligned}
$$




\section{K-THEORETiC DT theORY AND Hilbert SCHEMES}

The indices $\operatorname{ind}_{\sigma}\left(E_{\lambda,(-1,-1)}\left(t_{1}, t_{2}, t_{3}\right)\right)$ for preferred $\sigma$ can be computed from this expression.

By (4.13), we have

$$
\chi(\lambda,(-1,-1))=\frac{\left\|\lambda^{t}\right\|^{2}+\|\lambda\|^{2}}{2} .
$$

We conclude the following.

Proposition 4.2. The limits of the edge contribution $\hat{E}(\lambda,(-1,-1))$ under preferred slopes $\sigma$ are as follows:

\begin{tabular}{c|c}
$\sigma=\sigma\left(r_{1}, r_{2}, r_{3}\right)$ & $\hat{E}(\lambda,(-1,-1))\left(t_{1}, t_{2}, t_{3}\right)^{\sigma}$ \\
\hline$r_{1} \gg r_{2}>0 \gg r_{3}$ & $t^{\left\|\lambda^{t}\right\|^{2} / 2} q^{\|\lambda\|^{2} / 2}$ \\
\hline$r_{2} \gg r_{1}>0 \gg r_{3}$ & $t^{\left\|\lambda^{t}\right\|^{2} / 2} q^{\|\lambda\|^{2} / 2}$ \\
\hline$r_{1} \gg r_{3}>0 \gg r_{2}$ & $t^{\|\lambda\|^{2} / 2} q^{\left\|\lambda^{t}\right\|^{2} / 2}$ \\
\hline$r_{3} \gg r_{1}>0 \gg r_{2}$ & $t^{\|\lambda\|^{2} / 2} q^{\left\|\lambda^{t}\right\|^{2} / 2}$ \\
\hline$r_{2} \gg r_{3}>0 \gg r_{1}$ & $t^{\left\|\lambda^{t}\right\|^{2} / 2} q^{\|\lambda\|^{2} / 2}$ \\
\hline$r_{3} \gg r_{2}>0 \gg r_{1}$ & $t^{\|\lambda\|^{2} / 2} q^{\left\|\lambda^{t}\right\|^{2} / 2}$
\end{tabular}

The same argument applies to a $T$-invariant rational curve in $X$ with normal bundle $\mathcal{O} \oplus \mathcal{O}(-2)$, as depicted in Figure 7 .

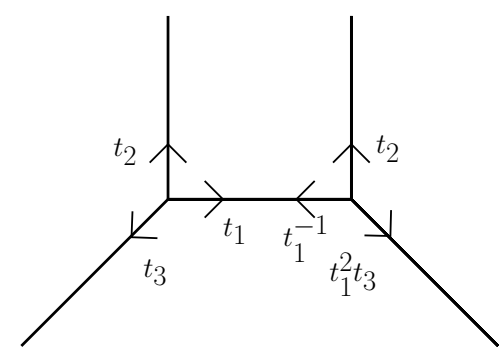

Figure 7: The weights along a $T$-invariant rational curve with normal bundle $\mathcal{O} \oplus \mathcal{O}(-2)$

By (4.7),

$$
\begin{aligned}
E_{\lambda,(0,-2)}\left(t_{1}, t_{2}, t_{3}\right)=\sum_{\square \in \lambda}( & \left(1+t_{1}^{-1}+\cdots+t_{1}^{-2 a(\square)}\right) t_{2}^{l(\square)+1} t_{3}^{-a(\square)} \\
& \left.-\left(t_{1}+\cdots+t_{1}^{2 a(\square)+1}\right) t_{2}^{-l(\square)} t_{3}^{a(\square)+1}\right) .
\end{aligned}
$$

By (4.13), we have

$$
\chi(\lambda,(0,-2))=|\lambda|+2 \frac{\|\left.\lambda\right|^{2}-|\lambda|}{2}=\| \lambda||^{2} .
$$

We conclude the following. 


\section{N. Arbesfeld}

Proposition 4.3. The limits of the edge contribution $\hat{E}(\lambda,(0,-2))$ under preferred slopes are as follows:

\begin{tabular}{c|c}
$\sigma=\sigma\left(r_{1}, r_{2}, r_{3}\right)$ & $\hat{E}(\lambda,(0,-2))\left(t_{1}, t_{2}, t_{3}\right)^{\sigma}$ \\
\hline$r_{1} \gg r_{2}>0 \gg r_{3}$ & $t^{\left(\|\lambda\|^{2}+|\lambda|\right) / 2} q^{\left(\|\lambda\|^{2}-|\lambda|\right) / 2}$ \\
\hline$r_{2} \gg r_{1}>0 \gg r_{3}$ & $t^{\|\lambda\|^{2}}$ \\
\hline$r_{1} \gg 0>r_{2} \gg r_{3}$ & $t^{\left(\|\lambda\|^{2}-|\lambda|\right) / 2} q^{\left(|| \lambda \|^{2}+|\lambda|\right) / 2}$ \\
\hline$r_{2} \gg 0>r_{1} \gg r_{3}$ & $t^{\|\lambda\|^{2}}$ \\
\hline$r_{3} \gg 0>r_{2} \gg r_{1}$ & $t^{\left(\|\lambda\|^{2}-|\lambda|\right) / 2} q^{\left(\|\lambda\|^{2}+|\lambda|\right) / 2}$ \\
\hline$r_{3} \gg 0>r_{1} \gg r_{2}$ & $q^{\|\lambda\|^{2}}$ \\
\hline$r_{3} \gg r_{2}>0 \gg r_{1}$ & $t^{\left(\|\lambda\|^{2}+|\lambda|\right) / 2} q^{\left(\|\lambda\|^{2}-|\lambda|\right) / 2}$ \\
\hline$r_{3} \gg r_{1}>0 \gg r_{2}$ & $q^{\|\lambda\|^{2}}$
\end{tabular}

4.3.3 Vertex limits. The vertex $V_{\pi}\left(t_{1}, t_{2}, t_{3}\right)$ defined in Section 4.2 is a quadratic expression in the character of the three-dimensional partition $\pi$. For an arbitrary partition $\pi$ and slope $\sigma$, there is no known concise expression for $\operatorname{ind}_{\sigma}\left(V_{\pi}\left(t_{1}, t_{2}, t_{3}\right)\right)$.

However, when $\sigma$ is a preferred slope, it is shown in [NO16, Section 8] that the index $\operatorname{ind}_{\sigma}\left(V_{\pi}\left(t_{1}, t_{2}, t_{3}\right)\right)$ is equal to the index of a different character written as a single sum over the boxes of $\pi$. This simpler expression for the index yields a combinatorial expression for preferred limits of the vertex that is well suited to computations.

We recall this procedure from [NO16, Section 8]. Some notation is needed. Fix a twodimensional partition $\nu \subset \mathbb{Z}_{\geqslant 0}^{2}$, and let $c_{i}^{-}$and $c_{i}^{+}$denote the ordered values of $b_{2}-b_{1}$ when $\left(b_{1}, b_{2}\right)$ are among the inner and outer corners of $\nu$, respectively; see Figure 8.

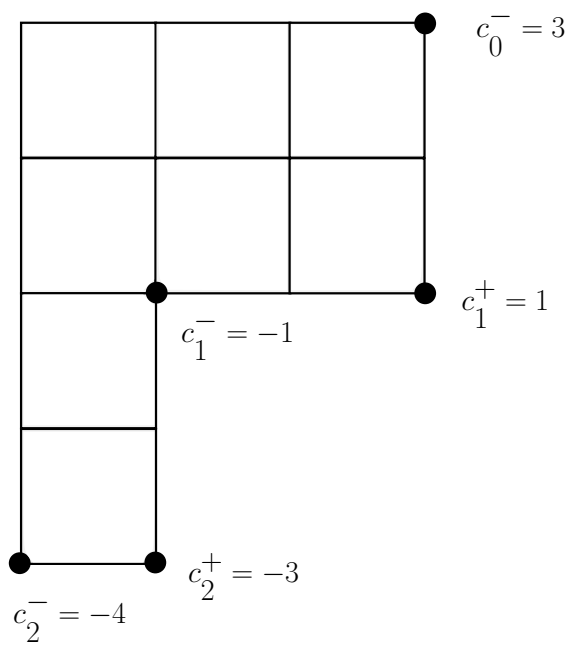

Figure 8: The partition $\lambda=(3,3,1,1)$ with corners labeled

To be precise, let $n_{1}>\cdots>n_{d(\nu)}$ be the distinct parts occurring in $\nu$ and $\nu$ be the partition 


\section{K-THEORETiC DT theORY AND Hilbert SCHEMES}

in which the part $n_{i}$ occurs with nonzero multiplicity $m_{i}$. Then for $i=0, \ldots, d(\nu)$, we set

$$
c_{i}^{-}=n_{i+1}-\sum_{j=1}^{i} m_{j}
$$

(here $\left.n_{d(\nu)}+1=0\right)$, and for $i=1, \ldots, d(\nu)$, we set

$$
c_{i}^{+}=n_{i}-\sum_{j=1}^{i} m_{j} .
$$

When $\nu=\varnothing$, the only corner is $c_{0}^{-}=0$.

Define a function $\psi_{\nu}: \mathbb{Z}_{\geqslant 0}^{3} \rightarrow\left\{t_{1}^{-1}, t_{2}^{-1}, t_{3}, t_{3}^{-1}\right\}$ as follows:

$$
\psi_{\nu}\left(b_{1}, b_{2}, b_{3}\right)= \begin{cases}t_{2}^{-1} & \text { if } b_{2}-b_{1}>c_{0}^{-} \\ & \text {or } c_{i}^{+}>b_{2}-b_{1}>c_{i}^{-} \text {for some } i \\ t_{1}^{-1} & \text { if } c_{i}^{-}>b_{2}-b_{1}>c_{i+1}^{+} \text {for some } i \\ & \text { or } c_{d(\nu)}^{-}>b_{2}-b_{1} \\ t_{3} & \text { if } b_{2}-b_{1}=c_{i}^{-} \text {for some } i \\ t_{3}^{-1} & \text { if } b_{2}-b_{1}=c_{i}^{+} \text {for some } i\end{cases}
$$

Now, given a three-dimensional partition $\pi$, a box $\square \in \pi$ and $k=1,2$ or 3 , set

$$
\delta_{k}(\square)= \begin{cases}1 & \text { if } \square \in \pi\langle k\rangle, \\ 0 & \text { if } \square \notin \pi\langle k\rangle .\end{cases}
$$

Define the virtual character $W_{\pi}\left(t_{1}, t_{2}, t_{3}\right)$ to be

$$
W_{\pi}\left(t_{1}, t_{2}, t_{3}\right)=\sum_{\square \in \pi}\left(\psi_{\pi^{(3)}}(\square)-\delta_{1}(\square) \psi_{\varnothing}(\square)-\delta_{2}(\square) \psi_{\varnothing}(\square)-\delta_{3}(\square) \psi_{\pi^{(3)}}(\square)\right) .
$$

Note the similarity between (4.12) and (4.22).

We can now recall from [NO16] a formula for $\operatorname{ind}_{\sigma}\left(V_{\pi}\right)$ for preferred slopes $\sigma$.

TheOREM 4.4 ([NO16, Theorem 2]). If $\pi$ is a three-dimensional partition and $\sigma\left(r_{1}, r_{2}, r_{3}\right)$ is a preferred slope with $\left|r_{1}\right|,\left|r_{2}\right| \gg\left|r_{3}\right|$, then

$$
\operatorname{ind}_{\sigma\left(r_{1}, r_{2}, r_{3}\right)}\left(V_{\pi}\left(t_{1}, t_{2}, t_{3}\right)\right)=\operatorname{ind}_{\sigma\left(r_{1}, r_{2}, r_{3}\right)}\left(W_{\pi}\left(t_{1}, t_{2}, t_{3}\right)-\kappa \cdot W_{\pi}^{\vee}\left(t_{1}, t_{2}, t_{3}\right)\right) .
$$

Given a preferred slope $\sigma\left(r_{1}, r_{2}, r_{3}\right)$ with $\left|r_{1}\right|,\left|r_{2}\right| \gg\left|r_{3}\right|$ and a two-dimensional partition $\nu$, define

$$
\Psi_{\nu}^{\sigma}: \mathbb{Z}_{\geqslant 0}^{3} \rightarrow\left\{-Q \kappa^{1 / 2},-Q \kappa^{-1 / 2}\right\}=\{t, q\}
$$

to be the function

$$
\Psi_{\nu}^{\sigma}\left(b_{1}, b_{2}, b_{3}\right)= \begin{cases}t & \text { if } \psi_{\nu}\left(b_{1}, b_{2}, b_{3}\right) \text { is attracting with respect to } \sigma \\ q & \text { if } \psi_{\nu}\left(b_{1}, b_{2}, b_{3}\right) \text { is repelling with respect to } \sigma .\end{cases}
$$

For $N>0$, set

$$
B_{N}=\left\{\left(b_{1}, b_{2}, b_{3}\right)|| b_{2}-b_{1} \mid \leqslant N\right\} .
$$

Combining (4.12) and Theorem 4.4, we obtain the following. 


\section{N. Arbesfeld}

Corollary 4.5. For a preferred slope $\sigma\left(r_{1}, r_{2}, r_{3}\right)$ with $\left|r_{1}\right|,\left|r_{2}\right| \gg\left|r_{3}\right|$ and $N \gg 0$, we have

$$
Q^{\chi(\pi)} \hat{a}\left(V_{\pi}\left(t_{1}, t_{2}, t_{3}\right)\right)^{\sigma\left(r_{1}, r_{2}, r_{3}\right)}=\frac{\prod_{\left\{\square \in \pi \cap B_{N} \mid \square \notin \pi\langle 3\rangle\right\}} \Psi_{\pi^{(3)}}^{\sigma}(\square)}{\prod_{\square \in B_{N} \cap \pi\langle 1\rangle} \Psi_{\varnothing}^{\sigma}(\square) \prod_{\square \in B_{N} \cap \pi\langle 2\rangle} \Psi_{\varnothing}^{\sigma}(\square)} .
$$

The limit $\hat{V}(\lambda, \mu, \nu)\left(t_{1}, t_{2}, t_{3}\right)^{\sigma}$ can be computed by summing (4.24) over all partitions $\pi \in$ $P(\lambda, \mu, \nu)$. We indicate how to do so when $r_{1} \gg r_{3}>0 \gg r_{2}$. Note that there are no values of $\left(r_{1}, r_{2}, r_{3}\right)$ and $N$ for which (4.24) holds simultaneously for all $\pi \in P(\lambda, \mu, \nu)$. However, for any fixed $m \in \mathbb{Z}$, one may choose $R_{m}, R_{m}^{\prime}$ and $N_{m} \in \mathbb{Z}$ such that for any $\pi \in P(\lambda, \mu, \nu)$ and triple $\left(r_{1}, r_{2}, r_{3}\right) \in \mathbb{Z}^{3}$ and $N>0$ satisfying

$$
\chi(\pi) \leqslant m, \quad r_{1}>R_{m}>R_{m}^{\prime}>r_{3}>0>r_{2} \quad \text { and } \quad N>N_{m},
$$

the equation (4.24) holds. In plainer language, any individual term of the limit

$$
\hat{V}(\lambda, \mu, \nu)\left(t_{1}, t_{2}, t_{3}\right)^{\sigma\left(r_{1}, r_{2}, r_{3}\right)}
$$

stabilizes as $r_{1} \gg r_{3}>0 \gg r_{2}$ and can be computed using (4.24).

Applying (4.24), we obtain

$$
\begin{aligned}
\hat{V} & (\lambda, \mu, \nu)\left(t_{1}, t_{2}, t_{3}\right)^{\sigma\left(r_{1}, r_{2}, r_{3}\right)} \\
& =\sum_{\pi \in P(\lambda, \mu, \nu)} \lim _{N \rightarrow \infty}\left(\frac{t^{-\left(|| \lambda^{t}||^{2}-|\lambda|\right) / 2} q^{-\left(\| \mu||^{2}-|\mu|\right) / 2}}{q^{N|\lambda|} t^{|\lambda|} t^{N|\mu|} t^{|\mu|}} \prod_{\left\{\square \in \pi \cap B_{N} \mid \square \notin \pi\langle 3\rangle\right\}} \Psi_{\nu}^{\sigma}(\square)\right) .
\end{aligned}
$$

A framework for evaluating such sums was introduced in [OR07]. Namely, given $\pi \in P(\lambda, \mu, \nu)$, the configuration $\pi / \pi\langle 3\rangle$ can be regarded as a sequence of two-dimensional partitions whose interlacing behavior is read off from $\nu$. Sums of the form (4.25) can be evaluated using a transfer matrix approach, where each such two-dimensional partition is assigned a weight using $\Psi_{\nu}^{\sigma}$.

This procedure is carried out in [IKV09, equation (150)] and yields the answer

$\sum_{\pi \in P(\lambda, \mu, \nu)} \lim _{N \rightarrow \infty} \frac{\prod_{\left\{\square \in \pi \cap B_{N}, \square \notin \pi\langle 3\rangle\right\}} \Psi_{\nu}^{\sigma}(\square)}{q^{N|\lambda| t^{|\lambda|} t^{N|\mu|} t^{|\mu|}}}=\frac{t^{-|\lambda| / 2} q^{-|\mu| / 2} \sum_{\eta}(q / t)^{|\eta| / 2} s_{\lambda / \eta}\left(t^{-\rho} q^{-\nu^{t}}\right) s_{\mu^{t} / \eta}\left(q^{-\rho} t^{-\nu}\right)}{\prod_{i, j \geqslant 0}\left(1-q^{i} t^{j+1}\right) \prod_{\square \in \nu}\left(1-q^{l(\square)} t^{a(\square)+1}\right)}$.

Here $t^{-\rho}$ denotes the sequence $\left(t^{1 / 2}, t^{3 / 2}, t^{5 / 2}, \ldots\right)$; the symbol $t^{-\lambda}$ denotes the sequence $\left(t^{-\lambda_{1}}\right.$, $\left.t^{-\lambda_{2}}, \ldots\right)$ and products of sequences are taken termwise. The $s_{\lambda / \eta}$ are skew Schur polynomials; see, for example, [IK06, Appendix A] for a concise introduction.

In particular,

$$
\hat{V}(\varnothing, \varnothing, \varnothing)\left(t_{1}, t_{2}, t_{3}\right)^{\sigma\left(r_{1}, r_{2}, r_{3}\right)}=\frac{1}{\prod_{i, j \geqslant 0}\left(1-q^{i} t^{j+1}\right)} .
$$

This equality also follows directly from Nekrasov's formula (3.2).

Incorporating the prefactor $t^{-\left(|| \lambda^{t}||^{2}-|\lambda|\right) / 2} q^{-\left(|| \mu||^{2}-|\mu|\right) / 2}$ from (4.24) and normalizing by (4.26), we set

$$
C(\lambda, \mu, \nu)(t, q)=\frac{t^{-\| \lambda^{t}||^{2} / 2} q^{-\|\mu\|^{2} / 2}}{\prod_{\square \in \nu}\left(1-q^{l(\square)} t^{a(\square)+1}\right)} \sum_{\eta}\left(\frac{q}{t}\right)^{|\eta| / 2} s_{\lambda / \eta}\left(t^{-\rho} q^{-\nu^{t}}\right) s_{\mu^{t} / \eta}\left(q^{-\rho} t^{-\nu}\right) .
$$




\section{K-THEORETIC DT THEORY AND Hilbert SCHEMES}

Up to a prefactor that is important for the computations in the following section, the series $C(\lambda, \mu, \nu)$ is equal to the refined topological vertex used in [IKV09].

The limits of $\hat{V}(\lambda, \mu, \nu)$ under other preferred slopes can be determined similarly or by using symmetries of three-dimensional partitions. We obtain the following.

Proposition 4.6. The limits of the normalized vertex contribution $\hat{V}^{\prime}(\lambda, \mu, \nu)$ under preferred slopes $\sigma$ are as follows:

\begin{tabular}{c|c}
$\sigma=\sigma\left(r_{1}, r_{2}, r_{3}\right)$ & $\hat{V}^{\prime}(\lambda, \mu, \nu)\left(t_{1}, t_{2}, t_{3}\right)^{\sigma}$ \\
\hline$r_{1} \gg r_{3}>0 \gg r_{2}$ & $C(\lambda, \mu, \nu)(t, q)$ \\
\hline$r_{1} \gg 0>r_{3} \gg r_{2}$ & $C\left(\mu^{t}, \lambda^{t}, \nu^{t}\right)(q, t)$ \\
\hline$r_{2} \gg r_{3}>0 \gg r_{1}$ & $C\left(\mu^{t}, \lambda^{t}, \nu^{t}\right)(t, q)$ \\
\hline$r_{2} \gg 0>r_{3} \gg r_{1}$ & $C(\lambda, \mu, \nu)(q, t)$
\end{tabular}

Note that there are four different choices of preferred slope corresponding to a given choice of "preferred direction" for the refined topological vertex.

\section{Dualities for tautological classes on $\left(\mathbb{C}^{2}\right)^{[n]}$}

We prove Theorem 1.2 and Proposition 1.3.

\subsection{Two toric geometries}

Let $X_{1}$ and $X_{2}$ be the smooth toric Calabi-Yau threefolds whose toric diagrams are those in Figure 9 , with the action of a three-dimensional torus $T=\operatorname{diag}\left(t_{1}, t_{2}, t_{3}\right)$ as indicated.

In Figure 10, we label each torus-invariant curve $C_{i}$ with its "Kähler parameter"; with regards to (2.4), this parameter denotes the variable $u^{\left[C_{i}\right]}$. Homologous curves in $X_{1}$ are assigned the same parameter.
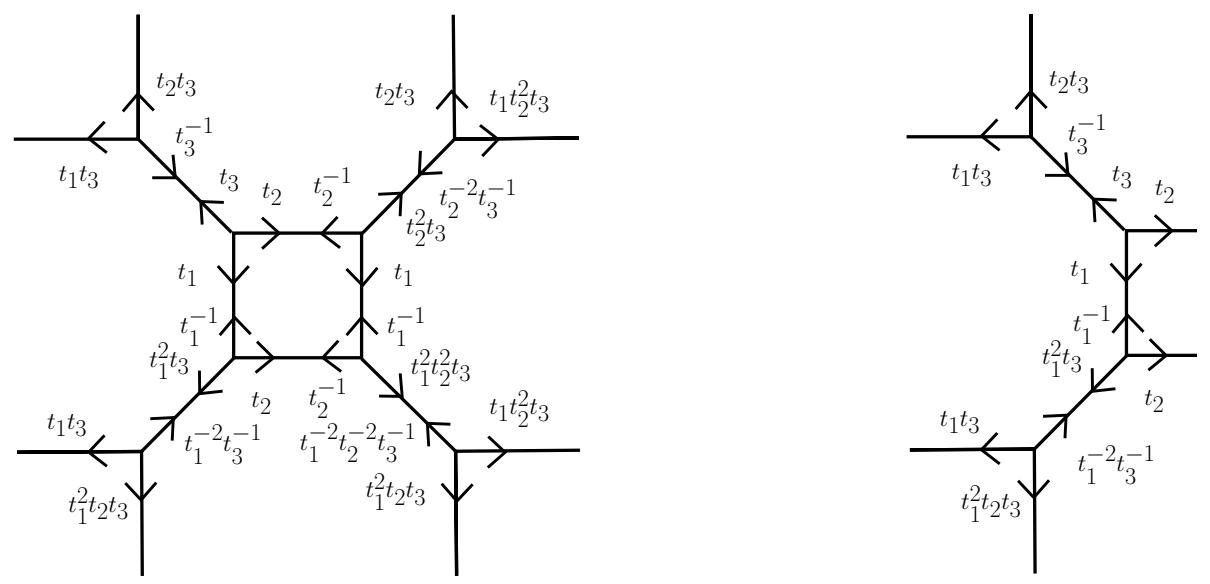

Figure 9: The Calabi-Yau threefolds $X_{1}$ and $X_{2}$, respectively, with $T$-weights labeled 


\section{N. Arbesfeld}
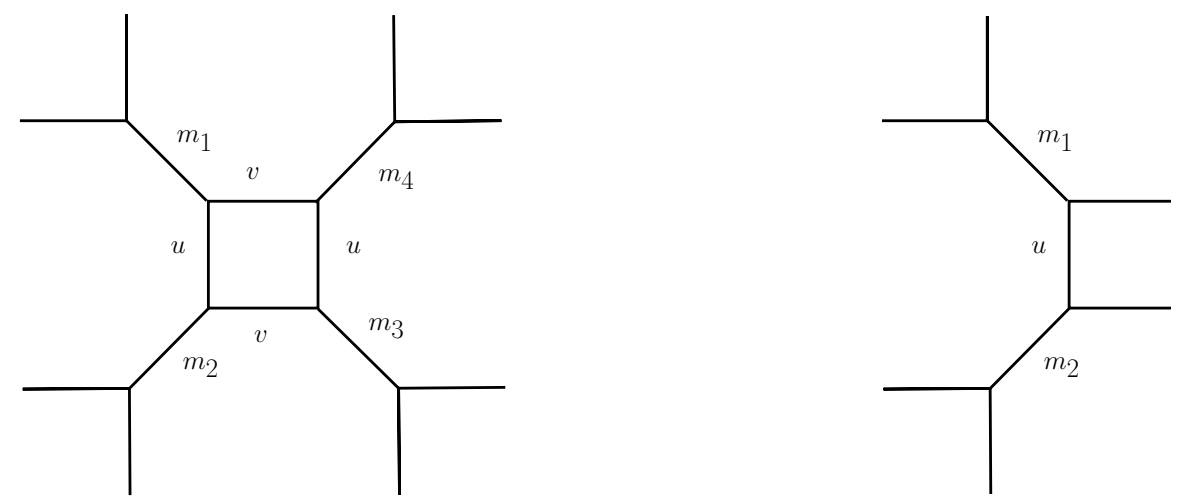

Figure 10: The Calabi-Yau threefolds $X_{1}$ and $X_{2}$, respectively, with Kähler parameters labeled

\subsection{Equality of limits}

Let $X$ denote either of $X_{1}$ or $X_{2}$. As before, let $\tilde{T}$ be the double cover of $T$ on which $\left(t_{1} t_{2} t_{3}\right)^{1 / 2}$ is defined, and let $A \subset T$ be the Calabi-Yau subtorus $\operatorname{ker}(\kappa)$; then $\tilde{T}$ acts on $X$ via the map $\tilde{T} \rightarrow T$.

For these two geometries, the possible noncompact directions of $X$ are those pointing vertically or horizontally in Figure 9; these are all of the form

$$
t_{1}^{i}\left(t_{1} t_{2} t_{3}\right)^{j / 2}, \quad t_{2}^{i}\left(t_{1} t_{2} t_{3}\right)^{j / 2} .
$$

Let $M$ be a component $\operatorname{DT}(X, \beta, n)$ of $\operatorname{DT}(X)$. By $(4.18)$, the function $\chi\left(M, \tilde{\mathcal{O}}^{\text {vir }}\right)$ is $A$ balanced.

Recall from (4.20) the definition of $\sigma\left(r_{1}, r_{2}, r_{3}\right)$. The weight $t_{1}^{i}\left(t_{1} t_{2} t_{3}\right)^{j / 2}$ is attracting with respect to $\sigma\left(r_{1}, r_{2}, r_{3}\right)$ if $i \cdot r_{1}>0$ and repelling if $i \cdot r_{1}<0$, while $t_{2}^{i}\left(t_{1} t_{2} t_{3}\right)^{j / 2}$ is attracting with respect to $\sigma$ if $i \cdot r_{2}>0$ and repelling if $i \cdot r_{2}<0$. We conclude that the attracting or repelling behavior of the noncompact directions of $X$ depends only on the signs of $r_{1}$ and $r_{2}$.

Hence, for $M$-generic $\sigma\left(r_{1}, r_{2}, r_{3}\right)$, Theorem 1.5 implies that the value of the limit

$$
\chi\left(M, \tilde{\mathcal{O}}^{\text {vir }}\right)^{\sigma\left(r_{1}, r_{2}, r_{3}\right)}
$$

depends only on the signs of $r_{1}$ and $r_{2}$. The same result holds when $\mathrm{DT}(X)$ is replaced by the Hilbert scheme of points. We conclude the following.

Corollary 5.1. For $r_{3} \gg r_{2}>0 \gg r_{1}$ and $s_{2} \gg 0>s_{1} \gg s_{3}$, we have

$$
Z_{\mathrm{DT}}^{\prime}(X)^{\sigma\left(r_{1}, r_{2}, r_{3}\right)}=Z_{\mathrm{DT}}^{\prime}(X)^{\sigma\left(s_{1}, s_{2}, s_{3}\right)} \in \mathbb{Q}\left[\Gamma, u^{\beta}\right] .
$$

Here, the integral domain $\mathbb{Q}\left[\Gamma, u^{\beta}\right]$ consists of power series in the Kähler variables with coefficients in $\Gamma$. In Section 5.3, we will also write certain elements of $\Gamma$ as rational functions in $q^{1 / 2}$ and $t^{1 / 2}$; the rational functions we write can be identified with elements of $\Gamma$ by expanding in positive powers of $q$ and $t$.

While sufficient to establish our results for Hilbert schemes, we remark that our notion of noncompact weight does not produce the strongest expected results of the form of Corollary 5.1. Namely, if one assumes the K-theoretic DT/stable pairs correspondence [NO16, equation (16)], then noncompact weights should be replaced by weights corresponding to directions in which curves in $X$ may escape to infinity. Such a reformulation would imply stronger versions of Corollary 5.1 for some threefolds not considered in this paper. For example, the space of effective 


\section{K-THEORETiC DT THEORY AND HilBert SCHEMES}

curves in $\mathcal{K}_{\mathbb{P}^{1} \times \mathbb{P}^{1}}$ is compact; the K-theoretic DT/stable pairs correspondence would then imply that, for generic $\sigma$, the limit $Z_{\mathrm{DT}}^{\prime}\left(\mathcal{K}_{\mathbb{P}^{1} \times \mathbb{P}^{1}}\right)^{\sigma}$ does not depend on $\sigma$.

\subsection{Evaluation of limits}

To apply Corollary 5.1, we evaluate the limits (5.1).

Divide the toric one-skeleton of $X_{1}$ into eight trivalent vertices and eight bounded edges. Propositions 4.2, 4.3 and 4.6 enable us to evaluate the corresponding limits of (4.17). Choose the counter-clockwise orientation for the one-skeleton of $\Delta\left(X_{1}\right)$. Let

$$
\lambda_{1}, \lambda_{2}, \mu_{1}, \mu_{2}, \kappa_{1}, \kappa_{2}, \kappa_{3}, \kappa_{4}
$$

be two-dimensional partitions assigned to the bounded edges as shown in Figure 11.

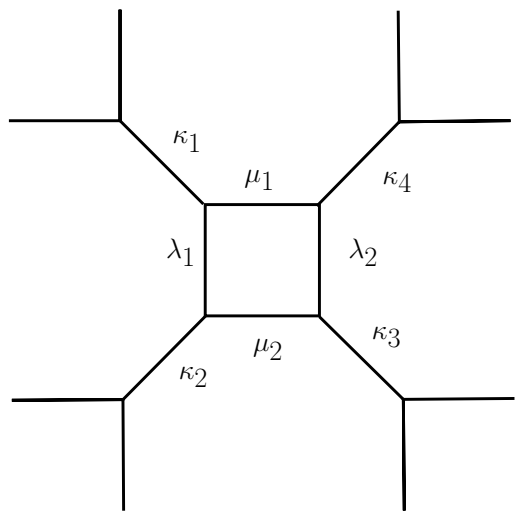

Figure 11: The Calabi-Yau threefold $X_{1}$ with two-dimensional partitions assigned to edges

Then, for $\sigma\left(r_{1}, r_{2}, r_{3}\right)$ with $r_{3} \gg r_{2}>0 \gg r_{1}$, the corresponding contribution to the series $Z_{\mathrm{DT}}^{\prime}\left(X_{1}\right)^{\sigma\left(r_{1}, r_{2}, r_{3}\right)}$ is the product of sixteen terms, one for each vertex and one for each edge. Reading the diagram left to right, then top to bottom, the edge terms are

$$
\begin{aligned}
& m_{1}^{\left|\kappa_{1}\right|} t^{\left\|\kappa_{1}^{t}\right\|^{2} / 2} q^{\left\|\kappa_{1}\right\|^{2} / 2}, \quad m_{4}^{\left|\kappa_{4}\right|} t^{|| \kappa_{4} \|^{2} / 2} q^{\left\|\kappa_{4}^{t}\right\|^{2} / 2}, \quad v^{\left|\mu_{1}\right|} q^{|| \mu_{1} \|^{2}}, \quad u^{\left|\lambda_{1}\right|} t^{\left(|| \lambda_{1}||^{2}+\left|\lambda_{1}\right|\right) / 2} q^{\left(|| \lambda_{1}||^{2}-\left|\lambda_{1}\right|\right) / 2}, \\
& u^{\left|\lambda_{2}\right|} t^{\left(|| \lambda_{2} \|^{2}-\left|\lambda_{2}\right|\right) / 2} q^{\left(|| \lambda_{2}||^{2}+\left|\lambda_{2}\right|\right) / 2}, \quad v^{\left|\mu_{2}\right|} t^{\left\|\mu_{2}\right\|^{2}}, \quad m_{2}^{\left|\kappa_{2}\right|} t^{\left\|\kappa_{2}^{t}\right\|^{2} / 2} q^{\left\|\kappa_{2}\right\|^{2} / 2}, \quad m_{3}^{\left|\kappa_{3}\right|} t^{\left\|\kappa_{3}\right\|^{2} / 2} q^{\left\|\kappa_{3}^{t}\right\|^{2} / 2},
\end{aligned}
$$

while the vertex terms are

$$
\begin{aligned}
& C\left(\kappa_{1}^{t}, \varnothing, \varnothing\right)(q, t)=q^{-\left\|\kappa_{1}\right\|^{2} / 2} s_{\kappa_{1}^{t}}\left(q^{-\rho}\right), \quad C\left(\varnothing, \kappa_{4}^{t}, \varnothing\right)(t, q)=q^{-\left\|\kappa_{4}^{t}\right\|^{2} / 2} s_{\kappa_{4}}\left(q^{-\rho}\right), \\
& C\left(\kappa_{1}, \lambda_{1}, \mu_{1}^{t}\right)(t, q)=\frac{t^{-\left\|\kappa_{1}^{t}\right\|^{2} / 2} q^{-\left\|\lambda_{1}\right\|^{2} / 2}}{\prod_{\square \in \mu_{1}^{t}} 1-t^{a(\square)+1} q^{l(\square)}} \sum_{\eta_{1}}\left(\frac{q}{t}\right)^{\left|\eta_{1}\right| / 2} s_{\kappa_{1} / \eta_{1}}\left(t^{-\rho} q^{-\mu_{1}}\right) s_{\lambda_{1}^{t} / \eta_{1}}\left(q^{-\rho} t^{-\mu_{1}^{t}}\right), \\
& C\left(\lambda_{2}^{t}, \kappa_{4}, \mu_{1}\right)(q, t)=\frac{q^{-\left\|\lambda_{2}\right\|^{2} / 2} t^{-\left\|\kappa_{4}\right\|^{2} / 2}}{\prod_{\square \in \mu_{1}} 1-q^{a(\square)+1} t^{l(\square)}} \sum_{\eta_{4}}\left(\frac{t}{q}\right)^{\left|\eta_{4}\right| / 2} s_{\lambda_{2}^{t} / \eta_{4}}\left(q^{-\rho} t^{-\mu_{1}^{t}}\right) s_{\kappa_{4}^{t} / \eta_{4}}\left(t^{-\rho} q^{-\mu_{1}}\right), \\
& C\left(\lambda_{1}^{t}, \kappa_{2}, \mu_{2}\right)(t, q)=\frac{t^{-\left\|\lambda_{1}\right\|^{2} / 2} q^{-\left\|\kappa_{2}\right\|^{2} / 2}}{\prod_{\square \in \mu_{2}} 1-t^{a(\square)+1} q^{l(\square)}} \sum_{\eta_{2}}\left(\frac{q}{t}\right)^{\left|\eta_{2}\right| / 2} s_{\lambda_{1}^{t} / \eta_{2}}\left(t^{-\rho} q^{-\mu_{2}^{t}}\right) s_{\kappa_{2}^{t} / \eta_{2}}\left(q^{-\rho} t^{-\mu_{2}}\right),
\end{aligned}
$$




\section{N. Arbesfeld}

$$
\begin{aligned}
& C\left(\kappa_{3}, \lambda_{2}, \mu_{2}^{t}\right)(q, t)=\frac{q^{-\left\|\kappa_{3}^{t}\right\|^{2} / 2} t^{-\left\|\lambda_{2}\right\|^{2} / 2}}{\prod_{\square \in \mu_{2}^{t}} 1-q^{a(\square)+1} t^{l(\square)}} \sum_{\eta_{3}}\left(\frac{t}{q}\right)^{\left|\eta_{3}\right| / 2} s_{\kappa_{3} / \eta_{3}}\left(q^{-\rho} t^{-\mu_{2}}\right) s_{\lambda_{2}^{t} / \eta_{3}}\left(t^{-\rho} q^{-\mu_{2}^{t}}\right), \\
& C\left(\varnothing, \kappa_{2}^{t}, \varnothing\right)(q, t)=t^{-\left\|\kappa_{2}^{t}\right\|^{2} / 2} s_{\kappa_{2}}\left(t^{-\rho}\right), \quad C\left(\kappa_{3}^{t}, \varnothing, \varnothing\right)(t, q)=t^{-\left\|\kappa_{3}\right\|^{2} / 2} s_{\kappa_{3}^{t}}\left(t^{-\rho}\right) .
\end{aligned}
$$

The limit $Z_{\mathrm{DT}}^{\prime}\left(X_{1}\right)^{\sigma\left(r_{1}, r_{2}, r_{3}\right)}$ is the sum over all possible choices of the two-dimensional partitions (5.2) of the product of these sixteen terms. To evaluate this sum, we use the methods of [IK06] for topological vertex computations. Their approach also works for the refined topological vertex. We use the following skew Schur polynomial identities which can be found, for example, in [Tak14]. For two sequences $x=\left(x_{0}, x_{1}, \ldots\right), y=\left(y_{0}, y_{1}, \ldots\right)$ and a formal variable $m$, set

$$
\{x, y\}_{m}=\prod_{i, j \geqslant 0}\left(1+m x_{i} y_{j}\right) .
$$

Then,

$$
\begin{aligned}
& \sum_{\lambda} u^{|\lambda|} s_{\lambda / \eta_{1}}\left(\left(x_{i}\right)\right) s_{\lambda / \eta_{2}}\left(\left(y_{j}\right)\right)=\frac{1}{\{x, y\}-u} \sum_{\lambda} u^{|\lambda|} s_{\eta_{1} / \lambda}\left(\left(u y_{j}\right)\right) s_{\eta_{2} / \lambda}\left(\left(u x_{i}\right)\right) \\
& \sum_{\lambda} u^{|\lambda|} s_{\lambda / \eta_{1}}\left(\left(x_{i}\right)\right) s_{\lambda^{t} / \eta_{2}}\left(\left(y_{j}\right)\right)=\{x, y\}_{u} \sum_{\lambda} u^{|\lambda|} s_{\eta_{1}^{t} / \lambda^{t}}\left(\left(u y_{j}\right)\right) s_{\eta_{2}^{t} / \lambda}\left(\left(u x_{i}\right)\right) .
\end{aligned}
$$

First, use (5.4) to sum over the partitions $\kappa_{i}$ labeling the outer edges. For $\kappa_{1}$, one has

$$
\sum_{\kappa_{1}} m_{1}^{\left|\kappa_{1}\right|} s_{\kappa_{1}^{t}}\left(q^{-\rho}\right) s_{\kappa_{1} / \eta_{1}}\left(t^{-\rho} q^{-\mu_{1}}\right)=\left\{t^{-\rho} q^{-\mu_{1}}, q^{-\rho}\right\}_{m_{1}} \cdot s_{\eta_{1}^{t}}\left(m_{1} q^{-\rho}\right),
$$

with analogous identities for the remaining $\kappa_{i}$.

Use the homogeneity of skew Schur polynomials to incorporate $(t / q)^{\left|\lambda_{1}\right| / 2_{-}}$and $(q / t)^{\left|\lambda_{2}\right| / 2_{-}}$ factors from the edge contributions and $(t / q)^{\left|\eta_{1}\right| / 2_{-}}$and $(q / t)^{\left|\eta_{3}\right| / 2}$-factors from the vertex contributions into the arguments of the skew Schur polynomials $s_{\lambda_{1}^{t} / \eta_{1}}$ and $s_{\lambda_{2}^{t} / \eta_{3}}$. For example, for the former one has

$$
(t / q)^{\left|\lambda_{1}\right| / 2}(q / t)^{\left|\eta_{1}\right| / 2} s_{\lambda_{1}^{t} / \eta_{1}}\left(q^{-\rho} t^{-\mu_{1}^{t}}\right)=s_{\lambda_{1}^{t} / \eta_{1}}\left(\sqrt{t / q} \cdot q^{-\rho^{-\mu_{1}^{t}}}\right) .
$$

Next, use (5.3) to sum over $\lambda_{1}$ and $\lambda_{2}$. For example, for $\lambda_{1}$ one obtains

$$
\begin{aligned}
\sum_{\lambda_{1}} u^{\left|\lambda_{1}\right|} s_{\lambda_{1}^{t} / \eta_{1}}\left(\sqrt{t / q} \cdot q^{-\rho} t^{-\mu_{1}^{t}}\right) s_{\lambda_{1}^{t} / \eta_{2}}\left(t^{-\rho} q^{-\mu_{2}^{t}}\right) \\
=\frac{\sum_{\lambda_{1}} u^{\left|\lambda_{1}\right|} s_{\eta_{2} / \lambda_{1}^{t}}\left(u \sqrt{t / q} \cdot q^{-\rho} t^{-\mu_{1}^{t}}\right) s_{\eta_{1} / \lambda_{1}^{t}}\left(u t^{-\rho} q^{-\mu_{2}^{t}}\right)}{\left\{\sqrt{t / q} \cdot q^{-\rho} t^{-\mu_{1}^{t}}, t^{-\rho} q^{-\mu_{2}^{t}}\right\}_{-u}} .
\end{aligned}
$$

Then, use (5.4) sum over the ancillary partitions $\eta_{i}$ associated with each vertex. For example, for $\eta_{1}$ one obtains

$$
\sum_{\eta_{1}} s_{\eta_{1}^{t}}\left(m_{1} q^{-\rho}\right) s_{\eta_{1} / \lambda_{1}^{t}}\left(u t^{-\rho} q^{-\mu_{2}^{t}}\right)=\left\{t^{-\rho} q^{-\mu_{2}^{t}}, q^{-\rho}\right\}_{m_{1} u} \cdot s_{\lambda_{1}}\left(m_{1} q^{-\rho}\right) .
$$

Finally, use (5.3) to again sum over $\lambda_{1}$ and $\lambda_{2}$. For example, for $\lambda_{1}$ one obtains

$$
\sum_{\lambda_{1}} u^{\left|\lambda_{1}\right|} s_{\lambda_{1}}\left(m_{1} q^{-\rho}\right) s_{\lambda_{1}}\left(m_{2} \sqrt{q / t} \cdot t^{-\rho}\right)=\frac{1}{\left\{\sqrt{q / t} \cdot t^{-\rho}, q^{-\rho}\right\}_{-u m_{1} m_{2}}} .
$$




\section{K-THEORETIC DT THEORY AND HilBerT SCHEMES}

Keeping careful track of prefactors, we obtain that for $r_{3} \gg r_{2}>0 \gg r_{1}$, the limit $Z_{\mathrm{DT}}^{\prime}\left(X_{1}\right)^{\sigma\left(r_{1}, r_{2}, r_{3}\right)}$ is

$$
\sum_{\mu_{1}, \mu_{2}} v^{\left|\mu_{1}\right|+\left|\mu_{2}\right|} \frac{q^{|| \mu_{1} \|^{2}} t^{\left\|\mu_{2}\right\|^{2}}}{V_{T}} \frac{V_{N}}{V_{D}}
$$

where

$$
\begin{aligned}
V_{T}= & \prod_{\square \in \mu_{1}}\left(1-t^{l(\square)} q^{a(\square)+1}\right)\left(1-t^{l(\square)+1} q^{a(\square)}\right) \prod_{\square \in \mu_{2}}\left(1-q^{l(\square)} t^{a(\square)+1}\right)\left(1-q^{l(\square)+1} t^{a(\square)}\right), \\
V_{N}= & \left\{t^{-\rho} q^{-\mu_{1}}, q^{-\rho}\right\}_{m_{1}}\left\{q^{-\rho} t^{-\mu_{2}}, t^{-\rho}\right\}_{m_{2}}\left\{q^{-\rho} t^{-\mu_{2}}, t^{-\rho}\right\}_{m_{3}}\left\{t^{-\rho} q^{-\mu_{1}}, q^{-\rho}\right\}_{m_{4}} \\
& \cdot\left\{t^{-\rho} q^{-\mu_{2}^{t}}, q^{-\rho}\right\}_{m_{1} u}\left\{q^{-\rho} t^{-\mu_{1}^{t}}, t^{-\rho}\right\}_{m_{2} u}\left\{q^{-\rho} t^{-\mu_{1}^{t}}, t^{-\rho}\right\}_{m_{3} u}\left\{t^{-\rho} q^{-\mu_{2}^{t}}, q^{-\rho}\right\}_{m_{4} u}, \\
V_{D}= & \left\{\sqrt{t / q} \cdot q^{-\rho} t^{-\mu_{1}^{t}}, t^{-\rho} q^{-\mu_{2}^{t}}\right\}_{-u}\left\{\sqrt{q / t} \cdot t^{-\rho} q^{-\mu_{2}^{t}}, q^{-\rho} t^{-\mu_{1}^{t}}\right\}_{-u} \\
& \cdot\left\{\sqrt{q / t} \cdot t^{-\rho}, q^{-\rho}\right\}_{-u m_{1} m_{2}}\left\{\sqrt{t / q} \cdot q^{-\rho}, t^{-\rho}\right\}_{-u m_{3} m_{4}} .
\end{aligned}
$$

Here, the factors of $V_{T}$ are expanded in positive powers of $t$ and $q$, and the factors of $V_{D}$ are expanded in positive powers of the Kahler variables. We record

$$
\left\{q^{-\rho} t^{-\lambda}, t^{-\rho}\right\}_{m}=\prod_{\square \in \lambda}\left(1+m \sqrt{q / t} \cdot q^{b_{1}} t^{-b_{2}}\right) \prod_{i, j \geqslant 0}\left(1+m q^{i+1 / 2} t^{j+1 / 2}\right) .
$$

All infinite products appearing in (5.5) are well defined as power series in the Kähler variables with coefficients in $\Gamma$.

The limit $Z_{\mathrm{DT}}^{\prime}\left(X_{1}\right)^{\sigma\left(s_{1}, s_{2}, s_{3}\right)}$ for $s_{2} \gg 0>s_{1} \gg s_{3}$ can be computed by the same procedure, yielding

$$
\sum_{\lambda_{1}, \lambda_{2}} u^{\left|\lambda_{1}\right|+\left|\lambda_{2}\right|} \frac{t^{\left\|\lambda_{1}\right\|^{2}} q^{\left\|\lambda_{2}\right\|^{2}}}{U_{T}} \frac{U_{N}}{U_{D}}
$$

where

$$
\begin{aligned}
U_{T}= & \prod_{\square \in \lambda_{1}}\left(1-q^{l(\square)} t^{a(\square)+1}\right)\left(1-q^{l(\square)+1} t^{a(\square)}\right) \prod_{\square \in \lambda_{2}}\left(1-t^{l(\square)} q^{a(\square)+1}\right)\left(1-t^{l(\square)+1} q^{a(\square)}\right), \\
U_{N}= & \left\{q^{-\rho} t^{-\lambda_{1}}, t^{-\rho}\right\}_{m_{1}}\left\{q^{-\rho} t^{-\lambda_{1}}, t^{-\rho}\right\}_{m_{2}}\left\{t^{-\rho} q^{-\lambda_{2}}, q^{-\rho}\right\}_{m_{3}}\left\{t^{-\rho} q^{-\lambda_{2}}, q^{-\rho}\right\}_{m_{4}} \\
& \cdot\left\{q^{-\rho} t^{-\lambda_{2}^{t}}, t^{-\rho}\right\}_{m_{1} v}\left\{q^{-\rho} t^{-\lambda_{2}^{t}}, t^{-\rho}\right\}_{m_{2} v}\left\{t^{-\rho} q^{-\lambda_{1}^{t}}, q^{-\rho}\right\}_{m_{3} v}\left\{t^{-\rho} q^{-\lambda_{1}^{t}}, q^{-\rho}\right\}_{m_{4} v},
\end{aligned}
$$

and

$$
\begin{aligned}
U_{D}= & \left\{\sqrt{q / t} \cdot t^{-\rho} q^{-\lambda_{1}^{t}}, q^{-\rho} t^{-\lambda_{2}^{t}}\right\}_{-v}\left\{\sqrt{t / q} \cdot q^{-\rho} t^{-\lambda_{2}^{t}}, t^{-\rho} q^{-\lambda_{1}^{t}}\right\}_{-v} \\
& \cdot\left\{\sqrt{t / q} \cdot q^{-\rho}, t^{-\rho}\right\}_{-v m_{1} m_{4}}\left\{\sqrt{q / t} \cdot t^{-\rho}, q^{-\rho}\right\}_{-v m_{2} m_{3}} .
\end{aligned}
$$

Again, all infinite products appearing in (5.7) are well defined.

Corollary 5.1 implies that (5.5) and (5.7) are equal. We perform specializations and substitutions to obtain Theorem 1.2.

First, we perform a specialization under which (5.5) and (5.7) each become sums over a single partition. Specialize

$$
m_{4}=-\sqrt{q / t} .
$$

Under (5.8), both (5.5) and (5.7) are well defined as elements of $\mathbb{Q}[\Gamma]\left[\left[u, v, m_{1}, m_{2}, m_{3}\right]\right]$. By (5.6), when $\lambda$ is nonempty, the infinite product $\left\{t^{-\rho} q^{-\lambda}, q^{-\rho}\right\}_{m}$ contains $(1+m \sqrt{t / q})$ as a factor. So, 


\section{N. Arbesfeld}

for nonempty $\lambda$, we have

$$
\left\{t^{-\rho} q^{-\lambda}, q^{-\rho}\right\}_{-\sqrt{q / t}}=0 .
$$

After implementing (5.8), it follows from (5.9) that only pairs of the form $\left(\varnothing, \mu_{2}\right)$ contribute to the sum (5.5).

Now, substitute

$$
m_{1}=-m_{1}^{\prime} \sqrt{t / q}, \quad m_{2}=-m_{2}^{\prime} \sqrt{t / q}, \quad m_{3}=-m_{3}^{\prime} \sqrt{t / q}, \quad u=u^{\prime} q / t, \quad v=v^{\prime} q / t .
$$

Having implemented (5.8) and (5.10), we apply (5.6) to the products $V_{T}$ and $V_{D}$ appearing in each summand of (5.5). After canceling like terms and keeping careful track of the remaining factors, we obtain that under (5.8) and (5.10), the sum (5.5) becomes

$$
\begin{aligned}
& \left(\sum_{\mu_{2}}\left(v^{\prime}\right)^{\left|\mu_{2}\right|} q^{\left|\mu_{2}\right|} t^{|| \mu_{2}||^{2}-\left|\mu_{2}\right|} \prod_{\square \in \mu_{2}} \frac{\left(1-m_{2}^{\prime} q^{b_{1}} t^{-b_{2}}\right)\left(1-m_{3}^{\prime} q^{b_{1}} t^{-b_{2}}\right)\left(1-u^{\prime} m_{1}^{\prime} q^{-b_{1}} t^{b_{2}}\right)}{\left(1-q^{l} t^{a+1}\right)\left(1-q^{l+1} t^{a}\right)\left(1-u^{\prime} q^{-b_{1}} t^{b_{2}}\right)}\right) \\
& \quad \cdot \prod_{i, j} \frac{\left(1-u^{\prime} m_{1}^{\prime} q^{i+1} t^{j}\right)\left(1-u^{\prime} m_{2}^{\prime} q^{i+1} t^{j}\right)}{\left(1-u^{\prime} q^{i+1} t^{j}\right)\left(1-u^{\prime} m_{1}^{\prime} m_{2}^{\prime} q^{i+1} t^{j}\right)} \\
& \quad \cdot \prod_{i, j}\left(1-q^{i+1} t^{j}\right)\left(1-m_{1}^{\prime} q^{i} t^{j+1}\right)\left(1-m_{2}^{\prime} q^{i} t^{j+1}\right)\left(1-m_{3}^{\prime} q^{i} t^{j+1}\right) .
\end{aligned}
$$

By a similar computation, under (5.8) and (5.10), the series (5.7) becomes

$$
\begin{aligned}
& \left(\sum_{\lambda_{1}}\left(u^{\prime}\right)^{\left|\lambda_{1}\right|} q^{\left|\lambda_{1}\right|} t^{|| \lambda_{1} \|^{2}-\left|\lambda_{1}\right|} \prod_{\square \in \lambda_{1}} \frac{\left(1-m_{1}^{\prime} q^{b_{1}} t^{-b_{2}}\right)\left(1-m_{2}^{\prime} q^{b_{1}} t^{-b_{2}}\right)\left(1-v^{\prime} m_{3}^{\prime} q^{-b_{1}} t^{b_{2}}\right)}{\left(1-q^{l} t^{a+1}\right)\left(1-q^{l+1} t^{a}\right)\left(1-v^{\prime} q^{-b_{1}} t^{b_{2}}\right)}\right) \\
& \quad \cdot \prod_{i, j} \frac{\left(1-v^{\prime} m_{2}^{\prime} q^{i+1} t^{j}\right)\left(1-v^{\prime} m_{3}^{\prime} q^{i+1} t^{j}\right)}{\left(1-v^{\prime} q^{i+1} t^{j}\right)\left(1-v^{\prime} m_{2}^{\prime} m_{3}^{\prime} q^{i+1} t^{j}\right)} \\
& \quad \cdot \prod_{i, j}\left(1-q^{i+1} t^{j}\right)\left(1-m_{1}^{\prime} q^{i} t^{j+1}\right)\left(1-m_{2}^{\prime} q^{i} t^{j+1}\right)\left(1-m_{3}^{\prime} q^{i} t^{j+1}\right) .
\end{aligned}
$$

Note that

$$
\prod_{\square \in \lambda} \frac{1}{\left(1-q^{l+1} t^{a}\right)\left(1-u^{\prime} q^{-b_{1}} t^{b_{2}}\right)}=\frac{q^{-|\lambda|} t^{-|| \lambda||^{2}+|\lambda|}}{\prod_{\square \in \lambda}\left(1-q^{-l-1} t^{-a}\right)\left(u^{\prime}-q^{b_{1}} t^{-b_{2}}\right)} .
$$

After canceling the factors common to (5.11) and (5.12), redistributing terms and using (5.13), we find that the expression

$$
\begin{aligned}
& \left(\sum_{\mu_{2}}\left(v^{\prime}\right)^{\left|\mu_{2}\right|} \prod_{\square \in \mu_{2}} \frac{\left(1-m_{2}^{\prime} q^{b_{1}} t^{-b_{2}}\right)\left(1-m_{3}^{\prime} q^{b_{1}} t^{-b_{2}}\right)\left(1-u^{\prime} m_{1}^{\prime} q^{-b_{1}} t^{b_{2}}\right)}{\left(1-q^{l} t^{a+1}\right)\left(1-q^{-l-1} t^{-a}\right)\left(u^{\prime}-q^{b_{1}} t^{-b_{2}}\right)}\right) \\
& \cdot \prod_{i, j} \frac{\left(1-v^{\prime} q^{i+1} t^{j}\right)\left(1-v^{\prime} m_{2}^{\prime} m_{3}^{\prime} q^{i+1} t^{j}\right)}{\left(1-v^{\prime} m_{2}^{\prime} q^{i+1} t^{j}\right)\left(1-v^{\prime} m_{3}^{\prime} q^{i+1} t^{j}\right)}
\end{aligned}
$$

is equal to the expression

$$
\begin{aligned}
& \left(\sum_{\lambda_{1}}\left(u^{\prime}\right)^{\left|\lambda_{1}\right|} \prod_{\square \in \lambda_{1}} \frac{\left(1-m_{1}^{\prime} q^{b_{1}} t^{-b_{2}}\right)\left(1-m_{2}^{\prime} q^{b_{1}} t^{-b_{2}}\right)\left(1-v^{\prime} m_{3}^{\prime} q^{-b_{1}} t^{b_{2}}\right)}{\left(1-q^{l} t^{a+1}\right)\left(1-q^{-l-1} t^{-a}\right)\left(v^{\prime}-q^{b_{1}} t^{-b_{2}}\right)}\right) \\
& \cdot \prod_{i, j} \frac{\left(1-u^{\prime} q^{i+1} t^{j}\right)\left(1-u^{\prime} m_{1}^{\prime} m_{2}^{\prime} q^{i+1} t^{j}\right)}{\left(1-u^{\prime} m_{1}^{\prime} q^{i+1} t^{j}\right)\left(1-u^{\prime} m_{2}^{\prime} q^{i+1} t^{j}\right)} .
\end{aligned}
$$




\section{K-THEORETiC DT THEORY AND HilBert SCHEMES}

By definition,

$$
\prod_{i, j} \frac{\left(1-v^{\prime} q^{i+1} t^{j}\right)\left(1-v^{\prime} m_{2}^{\prime} m_{3}^{\prime} q^{i+1} t^{j}\right)}{\left(1-v^{\prime} m_{2}^{\prime} q^{i+1} t^{j}\right)\left(1-v^{\prime} m_{3}^{\prime} q^{i+1} t^{j}\right)}=\operatorname{Sym}^{\bullet}\left(-v^{\prime} \frac{q\left(1-m_{2}^{\prime}\right)\left(1-m_{3}^{\prime}\right)}{(1-q)(1-t)}\right) .
$$

Regard (5.14) and (5.15) as power series in $u^{\prime}, v^{\prime}, m_{1}^{\prime}, m_{2}^{\prime}, m_{3}^{\prime}$ whose coefficients are rational functions in $q$ and $t$. Writing (5.14) and (5.15) in terms of $q^{-1}$ and $t$, we conclude that

$$
\frac{F\left(v^{\prime}, m_{2}^{\prime}, m_{3}^{\prime}, m_{1}^{\prime}, u^{\prime}\right)\left(q, t^{-1}\right)}{\operatorname{Sym}^{\bullet}\left(-v^{\prime} \frac{\left(1-m_{2}^{\prime}\right)\left(1-m_{3}^{\prime}\right)}{\left(1-q^{-1}\right)(1-t)}\right)}=\frac{F\left(u^{\prime}, m_{1}^{\prime}, m_{2}^{\prime}, m_{3}^{\prime}, v^{\prime}\right)\left(q, t^{-1}\right)}{\operatorname{Sym}^{\bullet}\left(-u^{\prime} \frac{\left(1-m_{1}^{\prime}\right)\left(1-m_{2}^{\prime}\right)}{\left(1-q^{-1}\right)(1-t)}\right)} .
$$

For now, assume Proposition 1.3, which asserts that

$$
\operatorname{Sym}^{\bullet}\left(-v^{\prime} \frac{\left(1-m_{2}^{\prime}\right)\left(1-m_{3}^{\prime}\right)}{\left(1-q^{-1}\right)(1-t)}\right)=F\left(v^{\prime}, m_{2}^{\prime}, m_{3}^{\prime}, m_{1}^{\prime}, 0\right)\left(q, t^{-1}\right) \text {. }
$$

We conclude that

$$
\frac{F\left(v^{\prime}, m_{2}^{\prime}, m_{3}^{\prime}, m_{1}^{\prime}, u^{\prime}\right)\left(q, t^{-1}\right)}{F\left(v^{\prime}, m_{2}^{\prime}, m_{3}^{\prime}, m_{1}^{\prime}, 0\right)\left(q, t^{-1}\right)}=\frac{F\left(u^{\prime}, m_{1}^{\prime}, m_{2}^{\prime}, m_{3}^{\prime}, v^{\prime}\right)\left(q, t^{-1}\right)}{F\left(u^{\prime}, m_{1}^{\prime}, m_{2}^{\prime}, m_{3}^{\prime}, 0\right)\left(q, t^{-1}\right)} .
$$

By definition, the expression $F\left(v, m_{2}, m_{3}, m_{1}, u\right)\left(q, t^{-1}\right)$ is also symmetric under the exchange of $m_{2}$ and $m_{3}$; this symmetry and (5.16) imply Theorem 1.2.

Proposition 1.3 is obtained from the same procedure applied to $X_{2}$, with Kähler parameters as labeled in Figure 10. Let $\lambda$ be the two-dimensional partition assigned to the bounded vertical edge of the diagram. Under the substitutions

$$
m_{1}=-m_{1}^{\prime} \sqrt{t / q}, \quad m_{2}=-m_{2}^{\prime} \sqrt{t / q}, \quad u=u^{\prime} q / t,
$$

the limit $Z_{\mathrm{DT}}^{\prime}\left(X_{2}\right)^{\sigma\left(r_{1}, r_{2}, r_{3}\right)}$ as $r_{3} \gg r_{2}>0 \gg r_{1}$ becomes

$$
\prod_{i, j} \frac{\left(1-m_{1}^{\prime} q^{i} t^{j+1}\right)\left(1-m_{2}^{\prime} q^{i} t^{j+1}\right)\left(1-m_{1}^{\prime} u^{\prime} q^{i+1} t^{j}\right)\left(1-m_{2}^{\prime} u^{\prime} q^{i+1} t^{j}\right)}{\left(1-u^{\prime} q^{i+1} t^{j}\right)\left(1-u^{\prime} m_{1}^{\prime} m_{2}^{\prime} q^{i+1} t^{j}\right)}
$$

while the limit $Z_{\mathrm{DT}}^{\prime}\left(X_{2}\right)^{\sigma\left(s_{1}, s_{2}, s_{3}\right)}$ as $s_{2} \gg 0>s_{1} \gg s_{3}$ becomes

$$
\begin{aligned}
& \left(\sum_{\lambda}\left(u^{\prime}\right)^{|\lambda|} q^{|\lambda|} t^{|| \lambda \|^{2}-|\lambda|} \prod_{\square \in \lambda} \frac{\left(1-m_{1}^{\prime} q^{b_{1}} t^{-b_{2}}\right)\left(1-m_{2}^{\prime} q^{b_{1}} t^{-b_{2}}\right)}{\left(1-q^{l} t^{a+1}\right)\left(1-q^{l+1} t^{a}\right)}\right) \\
& \cdot \prod_{i, j}\left(1-m_{1}^{\prime} q^{i} t^{j+1}\right)\left(1-m_{2}^{\prime} q^{i} t^{j+1}\right) .
\end{aligned}
$$

By Corollary 5.1, the series (5.17) and (5.18) are equal. We obtain the equality

$$
\sum_{\lambda}\left(u^{\prime}\right)^{|\lambda|} \prod_{\square \in \lambda} \frac{\left(1-m_{1}^{\prime} q^{b_{1}} t^{-b_{2}}\right)\left(1-m_{2}^{\prime} q^{b_{1}} t^{-b_{2}}\right)}{\left(1-q^{l} t^{a+1}\right)\left(1-q^{-l-1} t^{-a}\right)\left(-q^{b_{1}} t^{-b_{2}}\right)}=\operatorname{Sym} \bullet\left(u^{\prime} \frac{q\left(1-m_{1}^{\prime}\right)\left(1-m_{2}^{\prime}\right)}{(1-q)(1-t)}\right) ;
$$

here, we have also applied the $u^{\prime}=0$ specialization of the identity (5.13) to the series (5.18). Both sides of this identity are power series in $u^{\prime}, m_{1}^{\prime}, m_{2}^{\prime}$ whose coefficients are rational functions in $q$ and $t$.

It follows that

$$
F\left(u^{\prime}, m_{1}^{\prime}, m_{2}^{\prime}, m_{3}^{\prime}, 0\right)\left(q, t^{-1}\right)=\operatorname{Sym}^{\bullet}\left(-u^{\prime} \frac{\left(1-m_{1}^{\prime}\right)\left(1-m_{2}^{\prime}\right)}{\left(1-q^{-1}\right)(1-t)}\right),
$$

proving Proposition 1.3. 


\section{N. Arbesfeld}

\section{Tautological bundles on $S^{[n]}$}

Let $S$ be a complex projective smooth surface and $\mathcal{L}$ be a line bundle on $S$. In this section, we apply Theorem 1.2 and Proposition 1.3 to study the holomorphic Euler characteristics of certain Schur functors of the tautological bundles $\mathcal{L}^{[n]}$ on $S^{[n]}$.

\subsection{Reduction to toric surfaces}

Let $m, y$ and $z$ be formal parameters. Set

$$
\chi_{\Lambda}(S, \mathcal{L})=\sum_{k, n \geqslant 0} z^{n}(-m)^{k} \chi\left(S^{[n]}, \Lambda^{k} \mathcal{L}^{[n]}\right), \quad \chi_{\operatorname{Sym}}(S, \mathcal{L})=\sum_{k, n \geqslant 0} z^{n} y^{k} \chi\left(S^{[n]}, \operatorname{Sym}^{k} \mathcal{L}^{[n]}\right) .
$$

By the Hirzebruch-Riemann-Roch theorem, both $\chi_{\Lambda}$ and $\chi_{\text {Sym }}$ are of the form (1.5).

By [EGL01, Theorem 4.2], there exist universal series $A_{i} \in \mathbb{Q}[m, z]$, for $i=1, \ldots, 4$, such that

$$
\chi_{\Lambda}(S, \mathcal{L})=\exp \left(c_{1}(\mathcal{L})^{2} A_{1}+c_{1}(\mathcal{L}) c_{1}(S) A_{2}+c_{1}(S)^{2} A_{3}+c_{2}(S) A_{4}\right) .
$$

Set

$$
\gamma(S, \mathcal{L})=\left(c_{1}(\mathcal{L})^{2}, c_{1}(\mathcal{L}) c_{1}(S), c_{1}(S)^{2}, c_{2}(S)\right) \in \mathbb{Z}^{4}
$$

If $\gamma(S, L)=\sum_{i} a_{i} \gamma\left(S_{i}, \mathcal{L}_{i}\right)$ for $a_{i} \in \mathbb{Z}$, then

$$
\chi_{\Lambda}(S, \mathcal{L})=\prod_{i}\left(\chi_{\Lambda}\left(S_{i}, \mathcal{L}_{i}\right)\right)^{a_{i}} .
$$

Analogous results hold for $\chi_{\mathrm{Sym}}$.

We conclude that $\chi_{\Lambda}$ and $\chi_{\text {Sym }}$ can be determined for arbitrary $(S, \mathcal{L})$ by their values on any quadruple $\left\{\left(S_{i}, \mathcal{L}_{i}\right)\right\}_{i=1, \ldots, 4}$ for which the four vectors $\gamma\left(S_{i}, \mathcal{L}_{i}\right)$ are independent over $\mathbb{Q}$. Any set of generators $\left\{\left(S_{i}, \mathcal{L}_{i}\right)\right\}$ of the algebraic cobordism ring (see [LP09]) of surfaces equipped with a line bundle will satisfy this condition. In particular, such $\left(S_{i}, \mathcal{L}_{i}\right)$ can be chosen to be toric.

\subsection{Reduction to equivariant $\mathbb{C}^{2}$}

Let $S$ be a toric surface with the action of a two-dimensional algebraic torus $\operatorname{diag}\left(t_{1}, t_{2}\right)$ whose fixed locus is a finite set of points, and let $\mathcal{L}$ be an equivariant line bundle on $S$. Then, the series $\chi_{\Lambda}(S, \mathcal{L})$ and $\chi_{\mathrm{Sym}}(S, \mathcal{L})$ can be read off from the equivariant geometry near the fixed locus. Let $\left\{s_{i}\right\} \subset S$ be the torus-fixed points, let $U_{i}$ be a toric chart centered at $s_{i}$, let $w_{i_{1}}$ and $w_{i_{2}}$ denote the tangent weights at $s_{i}$, and let $l_{i}$ denote the torus weight of the fiber $\mathcal{L}_{s_{i}}$.

The torus action on $S$ induces an action on $S^{[n]}$ and, in particular, an action on $U_{i}^{[n]}$ for each $U_{i}$. The torus-fixed points of $S^{[n]}$ are given by products $\prod_{i} \mathcal{I}_{\lambda^{i}}$ of torus-fixed ideal sheaves $\mathcal{I}_{\lambda^{i}} \subset \mathcal{O}_{U_{i}}$ with $\sum\left|\lambda^{i}\right|=n$. At $\prod_{i} \mathcal{I}_{\lambda^{i}}$, the tangent space and the fiber of $\mathcal{L}^{[n]}$ have torus characters

$$
\sum_{i} T_{\lambda^{i}}\left(w_{i_{1}}, w_{i_{2}}\right), \quad \sum_{i} \sum_{\square \in \lambda^{i}} l_{i} w_{i_{1}}^{-b_{1}} w_{i_{2}}^{-b_{2}}
$$

respectively. Let $\mathcal{O}(l)$ denote the trivial bundle over $\mathbb{C}^{2}$ twisted by a torus weight $l$, and set $\chi_{\Lambda}\left(\mathbb{C}^{2}, \mathcal{O}(l)\right)\left(w_{1}, w_{2}\right)$ to be the character of

$$
\sum_{n, k \geqslant 0} z^{n}(-m)^{k} \chi\left(\mathbb{C}^{2[n]}, \Lambda^{k} \mathcal{O}(l)^{[n]}\right),
$$

where a torus acts on $\mathbb{C}^{2}$ by scaling the coordinate axes by weights $w_{1}, w_{2}$. Define $\chi_{\text {Sym }}\left(\mathbb{C}^{2}, \mathcal{O}(l)\right)$ 


\section{K-THEORETIC DT THEORY AND HilBerT SCHEMES}

analogously. Equivariant localization on $S$ implies

$$
\chi_{\Lambda}\left(\mathbb{C}^{2}, \mathcal{O}(l)\right)\left(w_{1}, w_{2}\right)=\sum_{\lambda} \frac{z^{|\lambda|}}{\Lambda^{\bullet}\left(T_{\lambda}^{\vee}\left(w_{1}, w_{2}\right)\right)} \prod_{\square \in \lambda}\left(1-m l w_{1}^{-b_{1}} w_{2}^{-b_{2}}\right),
$$

while equivariant localization on $S^{[n]}$ implies

$$
\chi_{\Lambda}(S, \mathcal{L})=\prod_{i} \chi_{\Lambda}\left(\mathbb{C}^{2}, \mathcal{O}\left(l_{i}\right)\right)\left(w_{i_{1}}, w_{i_{2}}\right) .
$$

The resulting identity is an equality of equivariant Euler characteristics. The nonequivariant series is recovered by specializing $t_{1}=t_{2}=1$.

\subsection{Exterior powers}

Using Proposition 1.3, we compute $\chi_{\Lambda}(S, \mathcal{L})$ for arbitrary $S$.

Corollary 6.1. For a projective surface $S$ with line bundle $\mathcal{L}$, we have

$$
\chi\left(S^{[n]}, \Lambda^{k} \mathcal{L}^{[n]}\right)=\left(\begin{array}{c}
n-k+\chi\left(\mathcal{O}_{S}\right)-1 \\
n-k
\end{array}\right)\left(\begin{array}{c}
\chi(\mathcal{L}) \\
k
\end{array}\right) .
$$

This result is a specialization of [Sca09, Theorem 5.2.1].

Proof. Let $F$ be as defined in (1.7). Proposition 1.3 and (2.3) imply that

$$
\chi_{\Lambda}\left(\mathbb{C}^{2}, \mathcal{O}(l)\right)\left(w_{1}, w_{2}\right)=F\left(z m l, \frac{1}{m l}, 0,0,0\right)\left(w_{1}, w_{2}\right)=\operatorname{Sym} \bullet\left(\frac{z-z m l}{\left(1-w_{1}^{-1}\right)\left(1-w_{2}^{-1}\right)}\right) .
$$

It suffices to check the corollary for toric $S$ and equivariant $\mathcal{L}$. Let $\left\{s_{i}\right\},\left\{w_{i_{1}}, w_{i_{2}}\right\}$ and $\left\{l_{i}\right\}$ be as in the previous section. Then, as equivariant series,

$$
\chi_{\Lambda}(S, \mathcal{L})=\prod_{i} \chi_{\Lambda}\left(\mathbb{C}^{2}, \mathcal{O}\left(l_{i}\right)\right)\left(w_{i_{1}}, w_{i_{2}}\right)=\operatorname{Sym} \bullet\left(\sum_{i} \frac{z-z m l_{i}}{\left(1-w_{i_{1}}^{-1}\right)\left(1-w_{i_{2}}^{-1}\right)}\right) .
$$

By equivariant localization, the expression (6.2) specializes under $t_{1}=t_{2}=1$ to

$$
\operatorname{Sym}^{\bullet}\left(z \chi\left(\mathcal{O}_{S}\right)-z m \chi(\mathcal{L})\right)=\frac{(1-z m)^{\chi(\mathcal{L})}}{(1-z)^{\chi\left(\mathcal{O}_{S}\right)}} .
$$

\subsection{Symmetric powers}

The author is not aware of a closed-form expression for the Euler characteristic of the $k$ th symmetric power of a tautological bundle in terms of $k$. Nonetheless, Theorem 1.2 and Proposition 1.3 can be applied to such symmetric powers.

We use Theorem 1.2 to rewrite $\chi_{\mathrm{Sym}}(S, \mathcal{L})$ as a fraction where the Segre variable $y$ enters the denominator in a controllable manner. Note that the series $\chi_{\mathrm{Sym}}\left(\mathbb{C}^{2}, \mathcal{O}(l)\right)\left(w_{1}, w_{2}\right)$ can be obtained by extracting all terms of the series $F(z, m, 0,0, y l)\left(w_{1}, w_{2}\right)$ of equal $z$-degree and $m$ degree. By Theorem 1.2, we have

$$
F(z, m, 0,0, y l)\left(w_{1}, w_{2}\right)=\frac{F(z, m, 0,0,0)\left(w_{1}, w_{2}\right)}{F(y l, 0,0, m, 0)\left(w_{1}, w_{2}\right)} \cdot F(y l, 0,0, m, z)\left(w_{1}, w_{2}\right) .
$$

By Proposition 1.3,

$$
F(z, m, 0,0,0)\left(w_{1}, w_{2}\right)=\operatorname{Sym} \bullet\left(\frac{z m-z}{\left(1-w_{1}^{-1}\right)\left(1-w_{2}^{-1}\right)}\right)
$$




\section{N. Arbesfeld}

$$
F(y l, 0,0, m, 0)\left(w_{1}, w_{2}\right)=\operatorname{Sym} \bullet\left(\frac{-y l}{\left(1-w_{1}^{-1}\right)\left(1-w_{2}^{-1}\right)}\right) .
$$

By definition,

$$
F(y l, 0,0, m, z)\left(w_{1}, w_{2}\right)=\sum_{\lambda} \frac{(y l)^{|\lambda|}}{\Lambda^{\bullet}\left(T_{\lambda}^{\vee}\left(w_{1}, w_{2}\right)\right)} \prod_{\square \in \lambda} \frac{1-m z w_{1}^{-b_{1}} w_{2}^{-b_{2}}}{z-w_{1}^{b_{1}} w_{2}^{b_{2}}} .
$$

Let $G\left(\mathbb{C}^{2}, \mathcal{O}(l)\right)\left(w_{1}, w_{2}\right)$ denote the series obtained by first extracting the terms of (6.6) of equal $z$-degree and $m$-degree, and then setting $m=1$; we have

$$
G\left(\mathbb{C}^{2}, \mathcal{O}(l)\right)\left(w_{1}, w_{2}\right)=\sum_{\lambda} \frac{(y l)^{|\lambda|}}{\Lambda^{\bullet}\left(T_{\lambda}^{\vee}\left(w_{1}, w_{2}\right)\right)} \prod_{\square \in \lambda} \frac{1-z w_{1}^{-b_{1}} w_{2}^{-b_{2}}}{-w_{1}^{b_{1}} w_{2}^{b_{2}}} .
$$

Consequently, extracting the terms of both sides of (6.3) of equal $z$-degree and $m$-degree, and then setting $m=1$, yields

$$
\chi_{\mathrm{Sym}}\left(\mathbb{C}^{2}, \mathcal{O}(l)\right)\left(w_{1}, w_{2}\right)=\operatorname{Sym} \bullet\left(\frac{z+y l}{\left(1-w_{1}^{-1}\right)\left(1-w_{2}^{-1}\right)}\right) G\left(\mathbb{C}^{2}, \mathcal{O}(l)\right)\left(w_{1}, w_{2}\right) .
$$

Suppose that $S$ is toric with equivariant line bundle $\mathcal{L}$, and let $\left\{s_{i}\right\},\left\{w_{i_{1}}, w_{i_{2}}\right\}$ and $\left\{l_{i}\right\}$ be as in Section 6.2. Then

$$
\begin{aligned}
\chi_{\operatorname{Sym}}(S, \mathcal{L}) & =\prod_{i} \chi_{\operatorname{Sym}}\left(\mathbb{C}^{2}, \mathcal{O}\left(l_{i}\right)\right)\left(w_{i_{1}}, w_{i_{2}}\right) \\
& =\operatorname{Sym} \bullet\left(\sum_{i} \frac{z+y l_{i}}{\left(1-w_{i_{1}}^{-1}\right)\left(1-w_{i_{2}}^{-1}\right)}\right) \prod_{i} G\left(\mathbb{C}^{2}, \mathcal{O}\left(l_{i}\right)\right)\left(w_{i_{1}}, w_{i_{2}}\right) .
\end{aligned}
$$

Set

$$
G(S, \mathcal{L})=\left.\left(\prod_{i} G\left(\mathbb{C}^{2}, \mathcal{O}\left(l_{i}\right)\right)\left(w_{i_{1}}, w_{i_{2}}\right)\right)\right|_{t_{1}=t_{2}=1}
$$

By equivariant localization, we conclude that

$$
\chi_{\mathrm{Sym}}(S, \mathcal{L})=\frac{1}{(1-z)^{\chi\left(\mathcal{O}_{S}\right)}(1-y)^{\chi(\mathcal{L})}} G(S, \mathcal{L}) .
$$

We apply (6.9). When $\chi\left(\mathcal{O}_{S}\right)=1$, we have the following stability result.

Corollary 6.2. Let $S$ be a projective surface with $\chi\left(\mathcal{O}_{S}\right)=1$, and let $\mathcal{L}$ be a line bundle on $S$. Then, for $n \geqslant k$, we have

$$
\chi\left(S^{[n]}, \operatorname{Sym}^{k} \mathcal{L}^{[n]}\right)=\left(\begin{array}{c}
\chi(\mathcal{L})+k-1 \\
k
\end{array}\right)
$$

in particular, the right-hand side does not depend on $n$.

Proof. It suffices to check the result for toric $S$ and equivariant $\mathcal{L}$. Fix $k \geqslant 0$, and set $G_{k}(S, \mathcal{L})$ to be the sum of all terms of $G(S, \mathcal{L})$ of $y$-degree at most $k$. As any monomial in $G(S, \mathcal{L})$ has $z$-degree no larger than $y$-degree, we may write

$$
G_{k}(S, \mathcal{L})=\sum_{j=0}^{k} g_{j}(S, \mathcal{L}) z^{j}
$$




\section{K-THEOREtic DT theORY AND Hilbert SCHEMES}

where $g_{j}(S, \mathcal{L}) \in \mathbb{Z}[y]\left(t_{1}, t_{2}\right)$. By $(6.9)$, given any $n \geqslant k$, the $z^{n} y^{k}$-term of $\chi_{\text {Sym }}(S, \mathcal{L})$ is the $y^{k}$-term of the expression

$$
\frac{1}{(1-y)^{\chi(\mathcal{L})}} \sum_{j=0}^{k} g_{j}(S, \mathcal{L})=\left.\frac{1}{(1-y)^{\chi(\mathcal{L})}} G_{k}(S, \mathcal{L})\right|_{z=1} .
$$

By (6.7), we have $\left.G\left(\mathbb{C}^{2}, \mathcal{O}(l)\right)\left(w_{1}, w_{2}\right)\right|_{z=1}=1$ for any $T$-weights $l, w_{1}$ and $w_{2}$. Therefore, $\left.G_{k}(S, \mathcal{L})\right|_{z=1}=1$.

When $\chi\left(\mathcal{O}_{S}\right) \neq 1$, the expressions (6.9) and (6.10) imply the following.

Corollary 6.3. For any $k$, the series $\sum_{n=0}^{\infty} z^{n} \chi\left(S^{[n]}, \mathrm{Sym}^{k} \mathcal{L}^{[n]}\right)$ is the Laurent expansion of a rational function in $z$ with denominator $(1-z)^{\chi\left(\mathcal{O}_{S}\right)}$.

We now compute the series $\chi_{\mathrm{Sym}}(S, \mathcal{L})$ up to $y$-degree 3 . The same approach can be used for any fixed degree in $y$.

Corollary 6.4. We have

$$
\begin{aligned}
& \chi\left(S^{[n]}, \mathcal{O}_{S}{ }^{[n]}\right)=\left(\begin{array}{c}
\chi\left(\mathcal{O}_{S}\right)+n-1 \\
n
\end{array}\right), \\
& \chi\left(S^{[n]}, \mathcal{L}^{[n]}\right)=\left(\begin{array}{c}
\chi\left(\mathcal{O}_{S}\right)+n-2 \\
n-1
\end{array}\right) \chi(\mathcal{L}), \\
& \chi\left(S^{[n]}, \operatorname{Sym}^{2} \mathcal{L}^{[n]}\right)=\left(\begin{array}{c}
\chi\left(\mathcal{O}_{S}\right)+n-3 \\
n-1
\end{array}\right) \chi\left(\mathcal{L}^{\otimes 2}\right)+\left(\begin{array}{c}
\chi\left(\mathcal{O}_{S}\right)+n-3 \\
n-2
\end{array}\right)\left(\begin{array}{c}
\chi(\mathcal{L})+1 \\
2
\end{array}\right), \\
& \chi\left(S^{[n]}, \operatorname{Sym}^{3} \mathcal{L}^{[n]}\right)=\left(\begin{array}{c}
\chi\left(\mathcal{O}_{S}\right)+n-3 \\
n-1
\end{array}\right) \chi\left(\mathcal{L}^{\otimes 3}\right) \\
& +\left(\begin{array}{c}
\chi\left(\mathcal{O}_{S}\right)+n-4 \\
n-2
\end{array}\right)\left(\chi\left(\mathcal{L}^{\otimes 2}\right) \chi(\mathcal{L})-\chi\left(\mathcal{L}^{\otimes 3} \otimes \mathcal{T} S^{\vee}\right)\right) \\
& +\left(\begin{array}{c}
\chi\left(\mathcal{O}_{S}\right)+n-4 \\
n-3
\end{array}\right)\left(\begin{array}{c}
\chi(\mathcal{L})+2 \\
3
\end{array}\right) \text {. }
\end{aligned}
$$

Proof. It again suffices to prove the result for toric $S$ and equivariant $\mathcal{L}$.

Computing the series $G\left(\mathbb{C}^{2}, \mathcal{O}(l)\right)$ of (6.7) explicitly up to $y$-degree 3 , one obtains

$$
\begin{aligned}
G & \left(\mathbb{C}^{2}, \mathcal{O}(l)\right)\left(t_{1}, t_{2}\right) \\
& =\operatorname{Sym} \bullet\left(\frac{-y l(1-z)+y^{2} l^{2}\left(z-z^{2}\right)+y^{3} l^{3}\left(z-z^{2}\right)\left(1-z\left(t_{1}^{-1}+t_{2}^{-1}\right)\right)}{\left(1-t_{1}^{-1}\right)\left(1-t_{2}^{-1}\right)}\right)+O\left(y^{4}\right) .
\end{aligned}
$$

Rewriting the right-hand side in terms of Euler characteristics of equivariant bundles over $\mathbb{C}^{2}$, we have

$$
\begin{aligned}
G\left(\mathbb{C}^{2}, \mathcal{O}(l)\right)\left(t_{1}, t_{2}\right)=\operatorname{Sym}^{\bullet}( & -y(1-z) \chi\left(\mathbb{C}^{2}, \mathcal{O}(l)\right)+y^{2}\left(z-z^{2}\right) \chi\left(\mathbb{C}^{2}, \mathcal{O}(l)^{\otimes 2}\right) \\
& \left.+y^{3}\left(z-z^{2}\right)\left(\chi\left(\mathbb{C}^{2}, \mathcal{O}(l)^{\otimes 3}\right)-z \chi\left(\mathbb{C}^{2}, \mathcal{O}(l)^{\otimes 3} \otimes \mathcal{T} \mathbb{C}^{2 \vee}\right)\right)\right)+O\left(y^{4}\right),
\end{aligned}
$$

where, on the right-hand side, a torus scales the coordinate axes of $\mathbb{C}^{2}$ by $t_{1}$ and $t_{2}$. 


\section{N. Arbesfeld}

By (6.8), (6.15) and equivariant localization, we conclude that

$$
\begin{aligned}
G(S, \mathcal{L})= & \operatorname{Sym}^{\bullet}\left(-y(1-z) \chi(S, \mathcal{L})+y^{2}\left(z-z^{2}\right) \chi\left(S, \mathcal{L}^{\otimes 2}\right)\right. \\
& \left.+y^{3}\left(z-z^{2}\right)\left(\chi\left(S, \mathcal{L}^{\otimes 3}\right)-z \chi\left(S, \mathcal{L}^{\otimes 3} \otimes \mathcal{T} S^{\vee}\right)\right)\right)+O\left(y^{4}\right) \\
= & \left(\frac{1-y}{1-z y}\right)^{\chi(\mathcal{L})}\left(\frac{1-z^{2} y^{2}}{1-z y^{2}}\right)^{\chi\left(\mathcal{L}^{\otimes 2}\right)}\left(\frac{1-z^{2} y^{3}}{1-z y^{3}}\right)^{\chi\left(\mathcal{L}^{\otimes 3}\right)}\left(\frac{1-z^{2} y^{3}}{1-z^{3} y^{3}}\right)^{\chi\left(\mathcal{L}^{\otimes 3} \otimes \mathcal{T} S^{\vee}\right)}+O\left(y^{4}\right) .
\end{aligned}
$$

The corollary then follows from (6.9).

The individual cohomology groups of bundles $\mathrm{Sym}^{k} \mathcal{L}^{[n]}$ have also been studied in connection with the strange duality conjecture for $\mathbb{P}^{2}$. Namely, equation (6.12) is shown in [EGL01, Proposition 5.6(ii)], and (6.13), (6.14) are consequences of [Sca15, Theorem 5.25]. In the cases when $n=2$ or 3, equation (6.13) is also a consequence of [Dan04, Theorems 1.1 and 1.2]. The approach of this section can also be used to determine values $\chi\left(S^{[n]}, \operatorname{Sym}^{k} \mathcal{L}_{1}^{[n]} \otimes \mathcal{D}_{\mathcal{L}_{2}}\right)$, as studied in [Dan04, Sca15]; here, given a line bundle $\mathcal{L}$ on $S$, the line bundle $\mathcal{D}_{\mathcal{L}}$ denotes the pullback under the Hilbert-Chow morphism of the bundle $\mathcal{L}^{\bigotimes n} / \mathfrak{S}^{n}$ on $\operatorname{Sym}^{n} S$.

\subsection{Rank two vector bundles on $S$}

As for line bundles, any rank $r$ vector bundle $\mathcal{V}$ on $S$ has an associated rank $r n$ tautological vector bundle $\mathcal{V}^{[n]}$ on $S^{[n]}$ whose fiber over $Y \in S^{[n]}$ is $H^{0}\left(\mathcal{O}_{Y} \otimes \mathcal{V}\right)$.

Let $\mathcal{V}$ denote a rank two vector bundle on $S$. Set

$$
\chi_{\Lambda}(S, \mathcal{V})=\sum_{n, k \geqslant 0} z^{n}(-m)^{k} \chi\left(S^{[n]}, \Lambda^{k} \mathcal{V}^{[n]}\right) .
$$

Again, there is no apparent concise closed formula for $\chi_{\Lambda}(S, \mathcal{V})$, but Theorem 1.2 can be used to compute the series up to any fixed degree in $m$.

Corollary 6.5. For a projective surface $S$ with rank two vector bundle $\mathcal{V}$, we have

$$
\begin{aligned}
& \chi\left(S^{[n]}, \mathcal{V}^{[n]}\right)=\left(\begin{array}{c}
\chi\left(\mathcal{O}_{S}\right)+n-2 \\
n-1
\end{array}\right) \chi(\mathcal{V}), \\
& \chi\left(S^{[n]}, \Lambda^{2} \mathcal{V}^{[n]}\right)=\left(\begin{array}{c}
\chi\left(\mathcal{O}_{S}\right)+n-3 \\
n-2
\end{array}\right)\left(\begin{array}{c}
\chi(\mathcal{V}) \\
2
\end{array}\right)+\left(\begin{array}{c}
\chi\left(\mathcal{O}_{S}\right)+n-3 \\
n-1
\end{array}\right) \chi\left(\Lambda^{2} \mathcal{V}\right) \\
& \chi\left(S^{[n]}, \Lambda^{3} \mathcal{V}^{[n]}\right)=\left(\begin{array}{c}
\chi\left(\mathcal{O}_{S}\right)+n-4 \\
n-3
\end{array}\right)\left(\begin{array}{c}
\chi(\mathcal{V}) \\
3
\end{array}\right)+\left(\begin{array}{c}
\chi\left(\mathcal{O}_{S}\right)+n-4 \\
n-2
\end{array}\right)\left(\chi(\mathcal{V}) \chi\left(\Lambda^{2} \mathcal{V}\right)-\chi\left(\mathcal{V} \otimes \Lambda^{2} \mathcal{V}\right)\right) .
\end{aligned}
$$

Proof. The argument of Sections 6.1 and 6.2 also applies to series of the form (1.5) when $\mathcal{L}$ is replaced by a higher-rank vector bundle. In particular, the series $\chi_{\Lambda}(S, \mathcal{V})$ is determined for arbitrary $(S, \mathcal{V})$ by its values at toric $S$ and equivariant $\mathcal{V}$.

Given torus weights $l_{1}, l_{2}$ and an action on $\mathbb{C}^{2}$ such that the coordinate axes are scaled by weights $w_{1}, w_{2}$, set

$$
\chi_{\Lambda}\left(\mathbb{C}^{2}, \mathcal{O}\left(l_{1}\right) \oplus \mathcal{O}\left(l_{2}\right)\right)\left(w_{1}, w_{2}\right)=\sum_{n, k \geqslant 0} z^{n}(-m)^{k} \chi\left(\left(\mathbb{C}^{2}\right)^{[n]}, \Lambda^{k}\left(\mathcal{O}\left(l_{1}\right) \oplus \mathcal{O}\left(l_{2}\right)\right)^{[n]}\right) .
$$

Then

$$
\chi_{\Lambda}\left(\mathbb{C}^{2}, \mathcal{O}\left(l_{1}\right) \oplus \mathcal{O}\left(l_{2}\right)\right)\left(t_{1}, t_{2}\right)=\left.F\left(z m l_{1}, \frac{1}{m l_{1}}, 0, \frac{m l_{2}}{y}, y\right)\left(t_{1}, t_{2}\right)\right|_{y=0}
$$




\section{K-THEORETIC DT THEORY AND HILBERT SCHEMES}

Note that the specialization $y=0$ on the right-hand side is well defined. By Theorem 1.2,

$$
\begin{aligned}
& F\left(z m l_{1}, \frac{1}{m l_{1}}, 0, \frac{m l_{2}}{y}, y\right)\left(t_{1}, t_{2}\right) \\
& \quad=\frac{F\left(z m l_{1}, \frac{1}{m l_{1}}, 0, \frac{m l_{2}}{y}, 0\right)\left(t_{1}, t_{2}\right)}{F\left(y, \frac{m l_{2}}{y}, 0, \frac{1}{m l_{1}}, 0\right)\left(t_{1}, t_{2}\right)} F\left(y, \frac{m l_{2}}{y}, 0, \frac{1}{m l_{1}}, z m l_{1}\right)\left(t_{1}, t_{2}\right) .
\end{aligned}
$$

By Proposition 1.3, we have

$$
\left.\frac{F\left(z m l_{1}, \frac{1}{m l_{1}}, 0, \frac{m l_{2}}{y}, 0\right)\left(t_{1}, t_{2}\right)}{F\left(y, \frac{m l_{2}}{y}, 0, \frac{1}{m l_{1}}, 0\right)\left(t_{1}, t_{2}\right)}\right|_{y=0}=\operatorname{Sym}_{y} \bullet\left(\frac{m l_{2}}{\left(1-t_{1}^{-1}\right)\left(1-t_{2}^{-1}\right)}\right) .
$$

Note that

$$
F\left(y, \frac{m l_{2}}{y}, 0, \frac{1}{m l_{1}}, z m l_{1}\right)\left(t_{1}, t_{2}\right)=\sum_{\lambda} \frac{\left(m l_{2}\right)^{|\lambda|}}{\Lambda^{\bullet} T_{\lambda}^{\vee}} \prod_{\square \in \lambda} \frac{\left(1-\frac{y}{m l_{2}} t_{1}^{-b_{1}} t_{2}^{-b_{2}}\right)\left(1-z t_{1}^{-b_{1}} t_{2}^{-b_{2}}\right)}{\left(1-z m l_{1} t_{1}^{-b_{1}} t_{2}^{-b_{2}}\right)} .
$$

In particular, each factor on the right-hand side of (6.19) is well defined under the specialization $y=0$, and only finitely many terms of (6.21) contribute to any fixed degree in $m$.

Expanding up to degree 3 in $m$ yields

$$
\begin{aligned}
F & \left.\left(y, \frac{m l_{2}}{y}, 0, \frac{1}{m l_{1}}, z m l_{1}\right)\left(t_{1}, t_{2}\right)\right|_{y=0} \\
& =\operatorname{Sym}^{\bullet}\left(\frac{m(1-z) l_{2}+m^{2}(1-z) z l_{1} l_{2}+m^{3}(1-z) z^{2}\left(l_{1}+l_{2}\right) l_{1} l_{2}}{\left(1-t_{1}^{-1}\right)\left(1-t_{2}^{-1}\right)}\right)+O\left(m^{4}\right) .
\end{aligned}
$$

Combining (6.20) and (6.22), we conclude that

$$
\begin{aligned}
\chi_{\Lambda}\left(\mathbb{C}^{2}, \mathcal{O}\left(l_{1}\right) \oplus \mathcal{O}\left(l_{2}\right)\right)\left(t_{1}, t_{2}\right) \\
=\operatorname{Sym} \bullet \\
\quad z \chi\left(\mathbb{C}^{2}, \mathcal{O}\right)-m z \chi\left(\mathbb{C}^{2}, \mathcal{O}\left(l_{1}\right) \oplus \mathcal{O}\left(l_{2}\right)\right)+m^{2}(1-z) z \chi\left(\mathbb{C}^{2}, \Lambda^{2}\left(\mathcal{O}\left(l_{1}\right) \oplus \mathcal{O}\left(l_{2}\right)\right)\right) \\
\left.\quad+m^{3}(1-z) z^{2} \chi\left(\mathbb{C}^{2},\left(\mathcal{O}\left(l_{1}\right) \oplus \mathcal{O}\left(l_{2}\right)\right) \otimes \Lambda^{2}\left(\mathcal{O}\left(l_{1}\right) \oplus \mathcal{O}\left(l_{2}\right)\right)\right)\right)+O\left(m^{4}\right)
\end{aligned}
$$

where, on the right-hand side, a torus scales the coordinate axes of $\mathbb{C}^{2}$ by $t_{1}$ and $t_{2}$.

Equivariant localization implies that

$$
\chi_{\Lambda}(S, \mathcal{V})=\frac{(1-z m)^{\chi(\mathcal{V})}}{(1-z)^{\chi\left(\mathcal{O}_{S}\right)}}\left(\frac{1-z^{2} m^{2}}{1-z m^{2}}\right)^{\chi\left(\Lambda^{2} \mathcal{V}\right)}\left(\frac{1-z^{3} m^{3}}{1-z^{2} m^{3}}\right)^{\chi\left(\mathcal{V} \otimes \Lambda^{2} \mathcal{V}\right)}+O\left(m^{4}\right)
$$

for all toric $S$ and equivariant $\mathcal{V}$. Note that (6.16) (and the same statement for $\mathcal{V}$ of arbitrary rank) also follows from (6.12).

It would be interesting if this paper's techniques could be applied to study Schur functors of other bundles over the Hilbert scheme of points on a surface and moduli of higher-rank sheaves. Examples of interest include the determinants of tautological bundles associated with higherrank vector bundles (which appear in the "Verlinde series" studied in [Joh20, MOP19]), tangent and cotangent bundles (which are relevant to the monopole branches of Vafa-Witten invariants studied in [Tho20]) and the Procesi bundle on the Hilbert scheme of points on $\mathbb{C}^{2}$. 


\section{N. Arbesfeld}

\section{ACKNOWLEDGEMENTS}

I am grateful to my thesis advisor Andrei Okounkov for his guidance and support, and to Rahul Pandharipande for asking a question that led to the present work. I thank Chiu-Chu Melissa Liu, Alina Marian, Davesh Maulik, Anton Mellit, Andrei Neguţ, Dragos Oprea, Petr Pushkar, Andrey Smirnov and Richard Thomas for helpful conversations and feedback. I also thank the anonymous referees for their careful attention and suggestions.

\section{REFERENCES}

AK13 H. Awata and H. Kanno, Changing the preferred direction of the refined topological vertex, J. Geom. Phys. 64 (2013), 91-110; doi:10.1016/j.geomphys.2012.10.014.

AKMV05 M. Aganagic, A. Klemm, M. Mariño and C. Vafa, The topological vertex, Comm. Math. Phys. 254 (2005), no. 2, 425-478; doi:10.1007/s00220-004-1162-z.

BF97 K. Behrend and B. Fantechi, The intrinsic normal cone, Invent. Math. 128 (1997), no. 1, 45-88; doi:10.1007/s002220050136.

CK09 I. Ciocan-Fontanine and M. Kapranov, Virtual fundamental classes via dg-manifolds, Geom. Topol. 13 (2009), no. 3, 1779-1804; doi:10.2140/gt.2009.13.1779.

Dan04 G. Danila, Sur la cohomologie de la puissance symétrique du fibré tautologique sur le schéma de Hilbert ponctuel d'une surface, J. Algebraic Geom. 13 (2004), no. 1, 81-113; doi:10.1090/ S1056-3911-03-00372-2.

EGL01 G. Ellingsrud, L. Göttsche and M. Lehn, On the cobordism class of the Hilbert scheme of a surface, J. Algebraic Geom. 10 (2001), no. 1, 81-100.

ES87 G. Ellingsrud and S. A. Strømme, On the homology of the Hilbert scheme of points in the plane, Invent. Math. 87 (1987), no. 2, 343-352; doi:10.1007/BF01389419.

FG10 B. Fantechi and L. Göttsche, Riemann-Roch theorems and elliptic genus for virtually smooth schemes, Geom. Topol. 14 (2010), no. 1, 83-115; doi:10.2140/gt.2010.14.83.

GK19 L. Göttsche and M. Kool, A rank 2 Dijkgraaf-Moore-Verlinde-Verlinde formula, Commun. Number Theory Phys. 13 (2019), no. 1, 165-201; doi:10.4310/CNTP.2019.v13.n1.a6.

GK20_ Virtual refinements of the Vafa-Witten formula, Comm. Math. Phys. 376 (2020), no. 1, 1-49; doi:10.1007/s00220-020-03748-7.

GP99 T. Graber and R. Pandharipande, Localization of virtual classes, Invent. Math. 135 (1999), no. 2, 487-518; doi:10.1007/s002220050293.

IK06 A. Iqbal and A.-K. Kashani-Poor, The vertex on a strip, Adv. Theor. Math. Phys. 10 (2006), no. 3, 317-343; doi:10.4310/ATMP.2006.v10.n3.a2.

IKV09 A. Iqbal, C. Kozçaz and C. Vafa, The refined topological vertex, J. High Energy Phys. 2009 (2009), no. 10, 069; doi:10.1088/1126-6708/2009/10/069.

Joh20 D. Johnson, Universal series for Hilbert schemes and strange duality, Int. Math. Res. Not. 2020 (2020), no. 10, 3130-3152; doi:10.1093/imrn/rny101.

Lee04 Y.-P. Lee, Quantum K-theory. I. Foundations, Duke Math. J. 121 (2004), no. 3, 389-424; doi:10.1215/S0012-7094-04-12131-1.

Leh99 M. Lehn, Chern classes of tautological sheaves on Hilbert schemes of points on surfaces, Invent. Math. 136 (1999), no. 1, 157-207; doi:10.1007/s002220050307.

LP09 M. Levine and R. Pandharipande, Algebraic cobordism revisited, Invent. Math. 176 (2009), no. 1, 63-130; doi:10.1007/s00222-008-0160-8.

Mel16 A. Mellit, Plethystic identities and mixed hodge structures of character varieties, 2016, arXiv: 1603.00193.

MNOP06 D. Maulik, N. Nekrasov, A. Okounkov and R. Pandharipande, Gromov-Witten theory and Donaldson-Thomas theory. I, Compos. Math. 142 (2006), no. 5, 1263-1285; doi:10.1112/ S0010437X06002302. 


\section{K-THEORETIC DT THEORY AND HilBERT SCHEMES}

MOP17 A. Marian, D. Oprea and R. Pandharipande, Segre classes and Hilbert schemes of points, Ann. Sci. Éc. Norm. Supér. (4) 50 (2017), no. 1, 239-267; doi:10.24033/asens. 2320.

MOP19 _ The combinatorics of Lehn's conjecture, J. Math. Soc. Japan 71 (2019), no. 1, 299-308; doi:10.2969/jmsj/78747874.

NO16 N. Nekrasov and A. Okounkov, Membranes and sheaves, Algebr. Geom. 3 (2016), no. 3, 320369; doi:10.14231/AG-2016-015.

Oko17 A. Okounkov, Lectures on K-theoretic computations in enumerative geometry, in Geometry of Moduli Spaces and Representation Theory, IAS/Park City Math. Ser., vol. 24 (Amer. Math. Soc., Providence, RI, 2017), 251-380; doi:10.1090/pcms/024.

Oko19 _ Takagi lectures on Donaldson-Thomas theory, Jpn. J. Math. 14 (2019), no. 1, 67-133; doi:10.1007/s11537-018-1744-8.

OR07 A. Okounkov and N. Reshetikhin, Random skew plane partitions and the Pearcey process, Comm. Math. Phys. 269 (2007), no. 3, 571-609; doi:10.1007/s00220-006-0128-8.

ORV06 A. Okounkov, N. Reshetikhin and C. Vafa, Quantum Calabi-Yau and classical crystals, in The Unity of Mathematics, Progr. Math., vol. 244 (Birkhäuser Boston, Boston, MA, 2006), 597-618; doi:10.1007/0-8176-4467-9_16.

PT09 R. Pandharipande and R.P. Thomas, Curve counting via stable pairs in the derived category, Invent. Math. 178 (2009), no. 2, 407-447; doi:10.1007/s00222-009-0203-9.

Qu18 F. Qu, Virtual pullbacks in K-theory, Ann. Inst. Fourier (Grenoble) 68 (2018), no. 4, 16091641; doi:10.5802/aif.3194.

Sca09 L. Scala, Cohomology of the Hilbert scheme of points on a surface with values in representations of tautological bundles, Duke Math. J. 150 (2009), no. 2, 211-267; doi:10.1215/ 00127094-2009-050.

Sca15_ Higher symmetric powers of tautological bundles on Hilbert schemes of points on a surface, 2015, arXiv:1502.07595.

Tak08 M. Taki, Refined topological vertex and instanton counting, J. High Energy Phys. 2008 (2008), no. 3, 048; doi:10.1088/1126-6708/2008/03/048.

Tak14 K. Takasaki, Remarks on partition functions of topological string theory on generalized conifolds, RIMS Kōkyūroku 1913 (2014), 182-201.

Tho92 R.W. Thomason, Une formule de Lefschetz en K-théorie équivariante algébrique, Duke Math. J. 68 (1992), no. 3, 447-462; doi:10.1215/S0012-7094-92-06817-7.

Tho00 R.P. Thomas, A holomorphic Casson invariant for Calabi-Yau 3-folds, and bundles on K3 fibrations, J. Differential Geom. 54 (2000), no. 2, 367-438; doi:10.4310/jdg/1214341649.

Tho20 _ Equivariant K-theory and refined Vafa-Witten invariants, Comm. Math. Phys. 378 (2020), no. 2, 1451-1500; doi:10.1007/s00220-020-03821-1.

Voi19 C. Voisin, Segre classes of tautological bundles on Hilbert schemes of surfaces, Algebr. Geom. 6 (2019), no. 2, 186-195; doi:10.14231/AG-2019-010.

WZ14 Z. Wang and J. Zhou, Tautological sheaves on Hilbert schemes of points, J. Algebraic Geom. 23 (2014), no. 4, 669-692; doi:10.1090/S1056-3911-2014-00623-8.

Noah Arbesfeld n.arbesfeld@imperial.ac.uk

Department of Mathematics, Imperial College London, London SW7 2AZ, United Kingdom 